\title{
The Atiyah-Singer Index Theorem as Passage to the Classical Limit in Quantum Mechanics
}

\author{
George A. Elliott ${ }^{1,2}$, Toshikazu Natsume ${ }^{3}$, Ryszard Nest ${ }^{1}$ \\ ${ }^{1}$ Mathematics Institute, Universitetsparken 5, DK-2100 Copenhagen $\varnothing$, Denmark \\ 2 Department of Mathematics, University of Toronto, Toronto, Ontario, Canada M5S 1A1 \\ ${ }^{3}$ Department of Mathematics, State University of New York at Buffalo, Buffalo, New York 14214, \\ USA
}

Received: 11 August 1995/Accepted: 5 December 1995

\begin{abstract}
A new approach to the Atiyah-Singer index theorem is described, using the technique of continuous fields of $C^{*}$-algebras. The proof is given in the case of elliptic pseudodifferential operators on $\mathbb{R}^{n}$.
\end{abstract}

\section{Introduction}

In the study of pseudodifferential operators (abbreviated $\Psi$ DOs) on a Euclidean space, or, more generally, on an open manifold, in order to extract information on the global properties of a $\Psi \mathrm{DO}$, one imposes "boundary" conditions. For instance, Seeley [S] investigated the class of elliptic $\Psi$ DOs of order 0 on $\mathbb{R}^{n}$ with the property, in a certain sense, of being equal to the identity at infinity. If $P$ belongs to this class, then its kernel and cokernel are finite-dimensional, and hence the (analytical) index,

$$
\text { index } P=\operatorname{dim} \operatorname{Ker} P-\operatorname{dim} \operatorname{Coker} P \text {, }
$$

is defined. For such operators, an index formula of Atiyah-Singer type has been established (see, for instance, [B-B]). Hörmander [H] also studied a class of $\Psi \mathrm{DOs}$ of order 0 on $\mathbb{R}^{n}$ and obtained an index formula. Bott and Seeley [B-S] and Callias [Ca] studied differential operators with coefficients and their derivatives decaying at infinity.

The classes of $\Psi$ DOs mentioned above cover a wide variety of operators. Nevertheless, interesting operators are left out of the picture (or at least not directly handled). For instance, in the one-dimensional case, the operator $D=x+\frac{d}{d x}$ is one of them. Not to mention that $D$ is an important operator in quantum mechanics.

In [E-N-N] we presented a new, simple, proof of the Connes Isomorphism Theorem for $C^{*}$-algebra crossed products by $\mathbb{R}$-actions, using continuous fields of $C^{*}$-algebras. As a byproduct, we obtained an index formula for the operator $D$ ([E-N-N, Remark 4.8]). In the present paper, we study a whole class of $\Psi$ DOs by similar methods, and establish an index formula (Theorem 3.1), describing the index in terms of the symbol.

Let us describe briefly the content of this paper. The general idea behind our proof is to consider the elements of $C_{0}\left(T^{*} \mathbb{R}^{n}\right)$ as the classical limit (as $\hbar$ goes to 
zero) of elements of the algebra of compact operators on $L^{2}\left(\mathbb{R}^{n}\right)$. More precisely, the two algebras are considered as quotients of the $C^{*}$ algebra of the Heisenberg group $H_{2 n+1}$, and the "limit" notion is provided by the topology of the spectrum of $H_{2 n+1}$. In the second section we introduce the class of pseudodifferential operators to which we apply this idea.

In the third section we state the main result of this paper, Theorem 3.1, which describes the index of an elliptic $\Psi \mathrm{DO}$ of positive order in terms of the topology of its symbol.

The fourth section contains construction of the analytic index of an elliptic operator $P$. The main point here is that the index is actually a $K_{0}$ class of the algebra of compact operators on $L^{2}\left(\mathbb{R}^{n}\right)$, and we describe a convenient way of representing it with the help of a graph projection $e$ of a closed operator associated with $P$.

The proof proper of the Theorem 3.1 is given in the section five. The strategy is fairly straightforward. In above terms, the graph projections $e_{\hbar}$ form a continuous field for $\hbar \in[0,1]$ (cf. Theorem 5.2), where the subscript refers to the operator with symbol scaled by $\hbar$, and where the limiting value $e_{0}$ is determined by the symbol of the operator $P$. The proof of this fact is the main analytic ingredient of this paper. The canonical trace on the algebra of compact operators gives rise to a field of cyclic cocycles for $\hbar>0$ which does not admit a continuous extension to $\hbar=0$. We replace it by a family of equivalent (in cyclic periodic cohomology) cyclic cocycles $\omega_{\hbar}$ which is actually continuous in $\hbar$ on the whole closed interval $[0,1]$. The rest of the proof consists of identifying the right hand side of the equality:

$$
\operatorname{index}(P)=\left\langle\omega_{\hbar}, e_{\hbar}\right\rangle=\left.\left\langle\omega_{\hbar}, e_{\hbar}\right\rangle\right|_{\hbar=0},
$$

where $\langle$,$\rangle denotes the pairing between K$-theory and cyclic cohomology.

In the last section we give an extension of the index theorem to the class of all (not necessarily positive order) elliptic operators on $\mathbb{R}^{n}$.

\section{Pseudodifferential operators on $\mathbb{R}^{n}$}

In the present paper, the Fourier transform of a function $u \in \mathscr{S}\left(\mathbb{R}^{n}\right)$ is given by the formula

$$
(F u)(\xi)=\int_{\mathbb{R}^{n}} e^{-i\langle x, \xi\rangle} u(x) d x,
$$

where $\langle x, \xi\rangle=x_{1} \xi_{1}+\cdots+x_{n} \xi_{n}$ and $d x=d x_{1} \cdots d x_{n}$, and the inverse Fourier transformation is defined by

$$
\left(F^{-1} v\right)(x)=(2 \pi)^{-n} \int_{\mathbb{R}^{n}} e^{i\langle x, \xi\rangle} v(\xi) d \xi,
$$

where $d \xi=d \xi_{1} \cdots d \xi_{n}$.

A pseudodifferential operator in $\mathbb{R}^{n}$ is an operator of the form

$$
(P u)(x)=(2 \pi)^{-n} \int_{\mathbb{R}^{n}} e^{i\langle x, \xi\rangle} a(x, \xi)(F u)(\xi) d \xi,
$$

where $u$ is a compactly supported $C^{\infty}$-function, and $a$ is a $C^{\infty}$-function on $\mathbb{R}^{n} \times \mathbb{R}^{n}$. In order for the formula above to make sense, the function $a$ has to satisfy certain growth conditions as $\xi$ approaches infinity. For instance, if $|a(x, \xi)| \rightarrow \infty$ as $\xi \rightarrow \infty$, 
then the growth rate must be polynomial. The choice of the asymptotic behaviour in the direction of $x$ is determined by the problems we are interested in.

In this paper we shall study the class of pseudodifferential operators investigated by M. Shubin in [Sh].

Definition 2.1 ([Sh, Def. 23.1]) The symbol class $\Gamma^{m}\left(\mathbb{R}^{n}\right), m \in \mathbb{R}$, consists of the $C^{\infty}$-functions $a$ on $\mathbb{R}^{n}$ such that for any multi-index $\alpha$, there exists a constant $C_{\alpha}$ with

$$
\left|\partial^{\alpha}(a)\right| \leqq C_{\alpha}\left(1+|z|^{2}\right)^{\frac{m-|x|}{2}}
$$

where $|z|^{2}=z_{1}^{2}+\cdots+z_{n}^{2}$ for $z=\left(z_{1}, \ldots, z_{n}\right) \in \mathbb{R}^{n}$. We shall say that $a \in \Gamma^{m}\left(\mathbb{R}^{n}\right)$ is of order $m$.

A symbol $a \in \Gamma^{m}\left(\mathbb{R}^{n} \times \mathbb{R}^{n}\right)$ defines an operator, denoted by $P_{a}$, or $\mathrm{Op}(a)$, by the formula,

$$
\begin{aligned}
\left(P_{a} u\right)(x) & =(2 \pi)^{-n} \int_{\mathbb{R}^{n}} e^{i\langle x, \xi\rangle} a(x, \xi)(F u)(\xi) d \xi \\
& =(2 \pi)^{-n} \int e^{i\langle x-y, \xi\rangle} a(x, \xi) u(y) d y d \xi
\end{aligned}
$$

$(x, \xi) \in \mathbb{R}^{n} \times \mathbb{R}^{n}, u \in C_{c}^{\infty}\left(\mathbb{R}^{n}\right)$.

The operator as above $P_{a}$ will be said to be of order $m$.

Let us summarize certain basic facts concerning the operators $P_{a}$. For more detail, see $[\mathrm{Sh}]$.

To begin with, $P_{a}$ extends to a continuous map

$$
P_{a}: \mathscr{S}\left(\mathbb{R}^{n}\right) \rightarrow \mathscr{S}\left(\mathbb{R}^{n}\right) .
$$

Here, of course, the space $\mathscr{S}\left(\mathbb{R}^{n}\right)$ of rapidly decreasing $C^{\infty}$-functions on $\mathbb{R}^{n}$ is equipped with the usual Fréchet space topology.

Convention. We will allways consider pseudodifferential operators as defined on $\mathscr{S}\left(\mathbb{R}^{n}\right)$.

Let $(\cdot, \cdot)$ denote the inner product in $L^{2}\left(\mathbb{R}^{n}\right)$ :

$$
(\phi, \psi)=\int_{\mathbb{R}^{n}} \phi(x) \overline{\psi(x)} d x, \quad \phi, \psi \in L^{2}\left(\mathbb{R}^{n}\right) .
$$

For each $a \in \Gamma^{m}\left(\mathbb{R}^{n} \times \mathbb{R}^{n}\right)$, there exists $a^{\prime} \in \Gamma^{m}\left(\mathbb{R}^{n} \times \mathbb{R}^{n}\right)$ such that

$$
\left(P_{a} \phi, \psi\right)=\left(\phi, P_{a^{\prime}} \psi\right), \quad \phi, \psi \in \mathscr{S}\left(\mathbb{R}^{n}\right) .
$$

That is, $P_{a}$ has a formal adjoint. Since $P_{a^{\prime}}: \mathscr{S}\left(\mathbb{R}^{n}\right) \rightarrow \mathscr{S}\left(\mathbb{R}^{n}\right)$ is also continuous, the operator $P_{a}$ extends to a continuous map $P_{a}: \mathscr{S}\left(\mathbb{R}^{n}\right)^{\prime} \rightarrow \mathscr{S}\left(\mathbb{R}^{n}\right)^{\prime}$, where $\mathscr{S}\left(\mathbb{R}^{n}\right)^{\prime}$ is the space of tempered distributions.

By definition, if $m \leqq m^{\prime}$, then $\Gamma^{m}\left(\mathbb{R}^{n}\right) \subseteq \Gamma^{m \prime}\left(\mathbb{R}^{n}\right)$. If $a \in \Gamma^{0}\left(\mathbb{R}^{n} \times \mathbb{R}^{n}\right)$, then $P_{a}: \mathscr{S}\left(\mathbb{R}^{n}\right) \rightarrow \mathscr{S}\left(\mathbb{R}^{n}\right)$ extends to a bounded operator on $L^{2}\left(\mathbb{R}^{n}\right)$. Moreover, if $a \in \Gamma^{m}\left(\mathbb{R}^{n} \times \mathbb{R}^{n}\right)$, and $m<0$, then $P_{a}$ is a compact operator on $L^{2}\left(\mathbb{R}^{n}\right)$. Set $\Gamma^{-\infty}=\bigcap_{m} \Gamma^{m}\left(\mathbb{R}^{n} \times \mathbb{R}^{n}\right)$. Then $\Gamma^{-\infty}=\mathscr{S}\left(\mathbb{R}^{n} \times \mathbb{R}^{n}\right)$ and, for any $a \in S^{-\infty}$, the operator $P_{a}$ is an integral operator with kernel in $\mathscr{S}\left(\mathbb{R}^{n} \times \mathbb{R}^{n}\right)$. In particular, the image of $P_{a}: \mathscr{S}\left(\mathbb{R}^{n}\right)^{\prime} \rightarrow \mathscr{S}\left(\mathbb{R}^{n}\right)^{\prime}$ is contained in $\mathscr{S}\left(\mathbb{R}^{n}\right)$. Such an operator is called infinitely smoothing. Let us now consider some examples. 
Example 2.4. Set $a(x, \xi)=x_{i}$. Then $\left(P_{a} \phi\right)(x)=x_{i} \phi(x), \phi \in \mathscr{S}\left(\mathbb{R}^{n}\right)$.

Remark 2.5. Usually, operators of pointwise multiplication by $C^{\infty}$-functions are considered to be operators of order 0 , but in our setting, they may have non-zero order. The above example is the case when the order of a multiplication operator is one.

Example 2.6. Consider the case $a(x, \xi)=\xi_{j}$. Then $P_{a}=\frac{1}{i} \frac{\partial}{\partial x_{j}}$, which is also of order 1 .

Let $a=\left(a_{i j}\right)$ be a $k \times k$ matrix of symbols of order $m$. Then the formula (2.3) makes sense if we replace $u$ by $V=\mathbb{C}^{k}$ valued functions. Thus we obtain an operator

$$
P_{a}: \mathscr{S}\left(\mathbb{R}^{n} ; V\right) \rightarrow \mathscr{S}\left(\mathbb{R}^{n} ; V\right),
$$

where $\mathscr{S}\left(\mathbb{R}^{n} ; V\right)$ is the space of rapidly decreasing $V$-valued functions. It is obvious that $P_{a}$ is given by the matrix $\left(P_{a_{l l}}\right)$. All the properties of pseudodifferential operators stated above also hold for $P_{a}$ with a matrix-valued symbol $a$. We shall call $a$ a $M_{k}$ valued symbol of order $m$.

Definition 2.7. $A M_{k}$-valued symbol a of order $m$ will be said to be elliptic if there exist positive constants $C$ and $R$ such that

$$
a(x, \xi)^{*} a(x, \xi) \geqq C\left(|x|^{2}+|\xi|^{2}\right)^{m} I_{k} \quad \text { for }|x|^{2}+|\xi|^{2} \geqq R
$$

where $I_{k}$ denotes the $k \times k$ identity matrix, $\geqq$ refers to the usual ordering of self-adjoint matrices and $*$ denotes the usual adjoint of matrices.

If the symbol $a$ is elliptic, then the operator $P_{a}$ will be said to be elliptic.

Example 2.8. Define $a \in C^{\infty}\left(\mathbb{R}^{n} \times \mathbb{R}^{n}\right)$ by $a(x, \xi)=x+i \xi$. Then $P_{a}=x+\frac{d}{d x}$, which is elliptic of order 1.

Example 2.9. For $(x, y, \xi, \eta) \in \mathbb{R}^{n} \times \mathbb{R}^{n}$, set

$$
a(x, y, \xi, \eta)=\left(\begin{array}{ll}
x+i y & i \xi-\eta \\
i \xi+\eta & x-i y
\end{array}\right) .
$$

Then $a$ is elliptic of order 1 , and

$$
P_{a}=\left(\begin{array}{cc}
x+i y & \frac{\partial}{\partial x}+i \frac{\partial}{\partial y} \\
\frac{\partial}{\partial x}-i \frac{\partial}{\partial y} & x-i y
\end{array}\right) .
$$

Example 2.10. The symbol $a(x, y, \xi, \eta)=x+i y+i \xi-\eta$ is not elliptic (in the sense of Definition 2.7).

Remark 2.11. Our definition of ellipticity (Definition 2.7) is strictly stronger than the usual definition (e.g., Definition 1.1 of [T, Chapter 3]). For instance, the operator of Example 2.10 is $x+i y+\frac{\partial}{\partial x}+i \frac{\partial}{\partial y}$, which is elliptic in the ordinary sense.

Example 2.12. Define a symbol $a$ by $a(x, \xi)=\left(1+\log \left(1+x^{2}+\xi^{2}\right)\right)^{-1}$. Then $a$ is of order 0 , but not of any order smaller than 0 . The symbol $a$ is not elliptic. 
In our setting, a fundamental role is played by non-local effects, i.e., the behaviour of functions as $|x| \rightarrow \infty$. Thus, we have to give up the property of being properly supported ([Sh]). On the other hand, the pseudodifferential operators studied in the present paper still have good properties, enjoyed by operators belonging to different classes, for instance the existence of a parametrix for any elliptic operator [Sh, Theorem 25.1]. Consequently, the regularity theorem holds [Sh, Corollary 25.1]. Furthermore, if $P_{a}$ is elliptic, then $P_{a}$ (as a possibly unbounded operator) on $L^{2}\left(\mathbb{R}^{n} ; V\right)$ is a Fredholm operator [Sh, Theorem 25.3]. We will denote by index $P_{a}$ the index of a Fredholm operator $P_{a}$.

If a pseudodifferential operator is elliptic, then the formal adjoint $P_{a}^{*}$ of $P_{a}$ is also elliptic [Sh, Lemma 25.1], and

$$
\text { index } P_{a}=\operatorname{dim} \operatorname{Ker} P_{a}-\operatorname{dim} \operatorname{Ker} P_{a}^{*} .
$$

Example 2.13 (2.8 continued). Consider the operator $P:=x+d / d x$. Then $P$ is elliptic, and index $P=1$. It is well known that the kernel of $P$ is spanned by the function $\phi(x)=e^{-\frac{x^{2}}{2}}$.

Example 2.14 Let $P$ denote the operator of Example 2.9. Then index $P=1$.

We conclude this section by recalling the following fact.

Theorem 2.15 ([Sh, Theorem 25.4]). Let $P$ be an elliptic operator of order $m>0$. If $P$ has a bounded inverse on $L^{2}\left(\mathbb{R}^{n} ; V\right)$, then $P^{-1}$ is the extension by continuity of a pseudodifferential operator of order $-m$.

Remark 2.3. Denote the operator of Remark 2.11 by $P$. The spectrum of $P^{*} P$ is discrete with infinite multiplicity, and so $P$ does not have index.

\section{The Index Theorem}

In this section we will formulate the main result, the proof will be given in Sect. 5 .

Once we know that if $P_{a}$ is elliptic, then $P_{a}$ has a Fredholm index (the analytic index), and our next goal is to describe index $P_{a}$ in terms of the topology of $a$ (the topological index).

As usual, we regard symbols as $C^{\infty}$-functions on $T^{*} \mathbb{R}^{n}=\mathbb{R}^{n} \times \mathbb{R}^{n}$. Suppose that $a$ is an $M_{k}$-valued elliptic symbol of order $m>0$. The operator of pointwise multiplication by the $C^{\infty}$-function $a$ is a closed operator on $L^{2}\left(T^{*} \mathbb{R}^{n} ; V\right)$. Let us denote this operator by $a$, and its adjoint by $a^{*}$.

Denote by $e_{a}$ the orthogonal projection of $L^{2}\left(T^{*} \mathbb{R}^{n} ; V\right) \oplus L^{2}\left(T^{*} \mathbb{R}^{n} ; V\right)$ onto the graph of $a$. This projection has the following matrix:

$$
e_{a}=\left(\begin{array}{cc}
\left(1+a^{*} a\right)^{-1} & \left(1+a^{*} a\right)^{-1} a^{*} \\
a\left(1+a^{*} a\right)^{-1} & a\left(1+a^{*} a\right)^{-1} a^{*}
\end{array}\right) .
$$

Ellipticity of $a$ implies that $\left(1+a^{*} a\right)^{-1},\left(1+a a^{*}\right)^{-1}$ are symbols of order $-2 m$, while $\left(1+a^{*} a\right)^{-1} a^{*}, a\left(1+a^{*} a\right)^{-1}$ are of order $-m$. In particular,

$$
\left(1+a^{*} a\right)^{-1},\left(1+a a^{*}\right)^{-1},\left(1+a^{*} a\right)^{-1} a^{*}, a\left(1+a^{*} a\right)^{-1} \in M_{k}\left(C_{0}\left(T^{*} \mathbb{R}^{n}\right)\right) .
$$


Since $1-a\left(1+a^{*} a\right)^{-1} a^{*}=\left(1+a a^{*}\right)^{-1}$, it follows that

$$
a\left(1+a^{*} a\right)^{-1} a^{*} \in M_{k}\left(C_{0}\left(T^{*} \mathbb{R}^{n}\right)^{\sim}\right),
$$

where $C_{0}\left(T^{*} \mathbb{R}^{n}\right)^{\sim}$ is the $C^{*}$-algebra $C_{0}\left(T^{*} \mathbb{R}^{n}\right)$ with unit adjoined.

Set $\hat{e}_{a}=e_{a}-\left(\begin{array}{ll}0 & 0 \\ 0 & 1\end{array}\right) \in M_{2 k}\left(C_{0}\left(T^{*} \mathbb{R}^{n}\right)\right)$. Since the entries of $\hat{e}_{a}$ are symbols of order $-m$, we see that

$$
\left|\operatorname{tr}\left(\hat{e}_{a}\left(d \hat{e}_{a}\right)^{2 n}\right)\right| \leqq C\left(1+|x|^{2}+|\xi|^{2}\right)^{\frac{-2 n-(2 n+1) m}{2}} \text { for some } C>0 .
$$

From the inequality $-2 n-(2 n+1) m<-2 n$, it follows that $\operatorname{tr}\left(\hat{e}_{a}\left(d \hat{e}_{a}\right)^{2 n}\right)$ is integrable on $T^{*} \mathbb{R}^{n}$.

The main result of this paper is stated as follows.

Theorem 3.1. If $P_{a}$ is an elliptic pseudodifferential operator of positive order, then

$$
\text { index } P_{a}=\frac{1}{(2 \pi i)^{n} n !} \int_{T^{*} \mathbb{R}^{n}} \operatorname{tr}\left(\hat{e}_{a}\left(d \hat{e}_{a}\right)^{2 n}\right)
$$

where $T^{*} \mathbb{R}^{n}$ is oriented by $d x_{1} \wedge d \xi_{1} \wedge \cdots \wedge d x_{n} \wedge d \xi_{n}>0$.

The proof will be given in Sect. 5 .

\section{The Analytic Index}

Let $P$ be an elliptic pseudodifferential operator of order $m>0$. We set $T_{0}=$ $P \mid C_{c}^{\infty}\left(\mathbb{R}^{n} ; V\right)$ and will regard $T_{0}$ as a densely defined unbounded operator on $L^{2}\left(\mathbb{R}^{n} ; V\right)$. Since $P$ has the formal adjoint $P^{*}$, the operator $T_{0}$ is closable. Let $T$ denote the closure of $T_{0}$. The operator $T^{*} T$ is densely defined and self-adjoint, its domain $T^{*} T$ contains $C_{c}^{\infty}\left(\mathbb{R}^{n} ; V\right)$, and

$$
T^{*} T\left|C_{c}^{\infty}\left(\mathbb{R}^{n} ; V\right)=P^{*} P\right| C_{c}^{\infty}\left(\mathbb{R}^{n} ; V\right) .
$$

Set $\left(P^{*} P\right)_{0}=P^{*} P \mid C_{c}^{\infty}\left(\mathbb{R}^{n} ; V\right)$. Then

$$
\left(P^{*} P\right)_{0} \subset T^{*} T
$$

and, since $\overline{\left(P^{*} P\right)_{0}}$ is self-adjoint,

$$
\overline{\left(P^{*} P\right)_{0}}=T^{*} T .
$$

By [Sh, Theorem 26.3] (and its generalization), the spectrum of $T^{*} T$ is discrete with finite multiplicities. Similarly, the spectrum of $T T^{*}$ is also discrete with finite multiplicities. In particular, the eigenvalue 0 (if it exists) is isolated in the spectra of $T^{*} T$ and $T T^{*}$.

Denote by $e$ the orthogonal projection of $L^{2}\left(\mathbb{R}^{n} ; V\right) \oplus L^{2}\left(\mathbb{R}^{n} ; V\right)$ onto the graph of the closed operator $T$. The projection $e$ can be explicitly written in terms of $T$ :

$$
e=\left(\begin{array}{cc}
\left(1+T^{*} T\right)^{-1} & \left(1+T^{*} T\right)^{-1} T^{*} \\
T\left(1+T^{*} T\right)^{-1} & T\left(1+T^{*} T\right)^{-1} T^{*}
\end{array}\right) .
$$

We shall call $e$ the graph projection of $P$. 
Since the spectrum of $T^{*} T$ is discrete and with finite multiplicities, $\left(1+T^{*} T\right)^{-\frac{1}{2}}$ is a compact operator on $L^{2}\left(\mathbb{R}^{n} ; V\right)$. An application of the polar decomposition of $T$ shows that $\left(1+T^{*} T\right)^{-\frac{1}{2}} T^{*}$ is bounded. Thus

$$
\left(1+T^{*} T\right)^{-1},\left(1+T^{*} T\right)^{-1} T^{*} \in \mathscr{K}\left(L^{2}\left(\mathbb{R}^{n} ; V\right)\right) .
$$

As for $T\left(1+T^{*} T\right)^{-1} T^{*}$, we have

$$
1-T\left(1+T^{*} T\right)^{-1} T^{*}=\left(1+T T^{*}\right)^{-1}
$$

and discreteness of the spectrum of $T T^{*}$ implies that

$$
T\left(1+T^{*} T\right)^{-1} T^{*} \in \mathscr{K}\left(L^{2}\left(\mathbb{R}^{n} ; V\right)\right)^{\sim} .
$$

Here, as usual, $\mathscr{K}\left(L^{2}\left(\mathbb{R}^{n} ; V\right)\right)^{\sim}$ denotes the $C^{*}$-algebra $\mathscr{K}\left(L^{2}\left(\mathbb{R}^{n} ; V\right)\right)$ with unit adjoined. Thus,

$$
e \in M_{2}\left(\mathscr{K}\left(L^{2}\left(\mathbb{R}^{n} ; V\right)\right)^{\sim}\right)
$$

and

$$
e-\left(\begin{array}{ll}
0 & 0 \\
0 & 1
\end{array}\right) \in M_{2}\left(\mathscr{K}\left(L^{2}\left(\mathbb{R}^{n} ; V\right)\right)\right)
$$

In particular the difference class

$$
[e]-\left[\left(\begin{array}{ll}
0 & 0 \\
0 & 1
\end{array}\right)\right]
$$

determines an element of $K_{0}\left(\mathscr{K}\left(L^{2}\left(\mathbb{R}^{n} ; V\right)\right)\right)$.

Denote by $\operatorname{Ker} P$ and $\operatorname{Ker} P^{*}$ the projections onto the kernel of $P$ and the kernel of $P^{*}$, respectively.

Theorem 4.1. In $K_{0}\left(\mathscr{K}\left(L^{2}\left(\mathbb{R}^{n} ; V\right)\right)\right)$,

$$
[e]-\left[\left(\begin{array}{ll}
0 & 0 \\
0 & 1
\end{array}\right)\right]=[\operatorname{Ker} P]-\left[\operatorname{Ker} P^{*}\right] .
$$

Proof. The curve

$$
[1, \infty) \ni \lambda \mapsto\left(\begin{array}{cc}
\left(1+\lambda^{2} T^{*} T\right)^{-1} & \left(1+\lambda^{2} T^{*} T\right)^{-1}\left(\lambda T^{*}\right) \\
\lambda T\left(1+\lambda^{2} T^{*} T\right)^{-1} & \lambda T\left(1+\lambda^{2} T^{*} T\right)^{-1} \lambda T^{*}
\end{array}\right)
$$

is norm continuous in $M_{2}\left(\mathscr{K}\left(L^{2}\left(\mathbb{R}^{n} ; V\right)\right)^{\sim}\right)$. Since the eigenvalue 0 is isolated in the spectrum, by the spectral theorem we have, as $\lambda \rightarrow \infty$, in the norm topology,

and

$$
\begin{aligned}
& \left(1+\lambda^{2} T^{*} T\right)^{-1} \rightarrow \operatorname{Ker} T^{*} T, \\
& \left(1+\lambda^{2} T^{*} T\right)^{-1}\left(\lambda T^{*}\right) \rightarrow 0
\end{aligned}
$$

$$
T\left(1+\lambda^{2} T^{*} T\right)^{-1} \lambda T^{*}=1-\left(1+\lambda^{2} T T^{*}\right)^{-1} \rightarrow 1-\operatorname{Ker} T T^{*} .
$$

Since $\operatorname{Ker} T^{*} T=\operatorname{Ker} T=\operatorname{Ker} P$ and $\operatorname{Ker} T T^{*}=\operatorname{Ker} T=\operatorname{Ker} P$, the conclusion follows. 
Corollary 4.2. Suppose that $P$ is elliptic of order $m>2 n$. Then the operators $\left(1+T^{*} T\right)^{-1}$ and $\left(1+T T^{*}\right)^{-1}$ are of trace class, and we have the equality

$$
\text { index } P=\operatorname{Tr}\left(\left(1+T^{*} T\right)^{-1}\right)-\operatorname{Tr}\left(\left(1+T T^{*}\right)^{-1}\right),
$$

where $\operatorname{Tr}$ is the canonical (normal, semifinite) trace on $\mathscr{K}\left(L^{2}\left(\mathbb{R}^{n} ; V\right)\right)$.

Proof. By [Sh, Lemma 25.1], the operators $1+T^{*} T$ and $1+T T^{*}$ are elliptic. Hence by Theorem 2.15, $\left(1+T^{*} T\right)^{-1}$ and $\left(1+T T^{*}\right)^{-1}$ are elliptic operators of order $-2 m$. Therefore, by [Sh, Proposition 27.2], these operators are of trace class.

The operators $\left(1+T^{*} T\right)^{-1} T^{*}$ and $T\left(1+T^{*} T\right)^{-1}$ are elliptic of order $-m<$ $-2 n$. Hence, again by [Sh, Proposition 27.2], $\left(1+T^{*} T\right)^{-1} T^{*}$ and $T\left(1+T^{*} T\right)^{-1}$ are of trace class.

Consider the trace class ideal $\mathcal{L}_{1}\left(L^{2}\left(\mathbb{R}^{n} ; V\right)\right)$ of $\mathscr{K}\left(L^{2}\left(\mathbb{R}^{n} ; V\right)\right)$. The observation above means that $e \in M_{2}\left(\mathcal{L}_{1}\left(L^{2}\left(\mathbb{R}^{n} ; V\right)\right)\right)$. Theorem 4.1 , together with the fact that $\mathcal{L}_{1}\left(L^{2}\left(\mathbb{R}^{n} ; V\right)\right)$ is stable under the holomorphic functional calculus, implies that

$$
[e]-\left[\left(\begin{array}{ll}
0 & 0 \\
0 & 1
\end{array}\right)\right]=[\operatorname{Ker} P]-\left[\operatorname{Ker} P^{*}\right]
$$

in $K_{0}\left(\mathcal{L}_{1}\left(L^{2}\left(\mathbb{R}^{n} ; V\right)\right)\right)$. By evaluating the trace $\operatorname{Tr}$, we get that

$$
\begin{aligned}
\operatorname{Tr}\left(\left(1+T^{*} T\right)^{-1}\right)-\operatorname{Tr}\left(\left(1+T T^{*}\right)^{-1}\right) & =\operatorname{Tr}(\operatorname{Ker} P)-\operatorname{Tr}\left(\operatorname{Ker} P^{*}\right) \\
& =\operatorname{index} P .
\end{aligned}
$$

\section{Proof of the Index Theorem}

The proof will be based on an idea introduced in [E-N-N]. Consider the family $\mathscr{A}^{\prime}=\left(A^{\prime}(\hbar)\right)$ of $C^{*}$-algebras parametrized by the interval $[0,1]$ defined by

$$
\begin{aligned}
& A^{\prime}(0)=C_{0}\left(T^{*} \mathbb{R}^{n}\right), \\
& A^{\prime}(\hbar)=\mathscr{K}\left(L^{2}\left(\mathbb{R}^{n}\right)\right), \quad \hbar>0 .
\end{aligned}
$$

We can furnish $\mathscr{A}^{\prime}$ with a structure of continuous field. As in [E-N-N] we make use of the irreducible unitary representations $\left(\pi_{\hbar}\right)$ of the $(2 n+1)$-dimensional Heisenberg group $H_{2 n+1}$. Write $[y, x, z]$ for the element

$$
\left(\begin{array}{ccc}
1 & x & z \\
& \ddots & y \\
0 & & 1
\end{array}\right) \in H_{2 n+1} .
$$

We use the Haar measure $\frac{1}{(2 \pi)^{2 n+1}} d x d y d z$ on $H_{2 n+1}$. The representation $\pi_{\hbar}$ on $L^{2}\left(\mathbb{R}^{n}\right)$ is given by

$$
\pi_{\hbar}([y, x, z]) \phi(t)=e^{i(\hbar z+y \cdot t)} \phi(t+\hbar x), \quad \phi \in L^{2}\left(\mathbb{R}^{n}\right) .
$$


Let $f \in \mathscr{S}\left(T^{*} \mathbb{R}^{n} \times \mathbb{R}\right)$. Regard the Fourier transform $F f$ of $f$ as an element of $\mathscr{S}\left(H_{2 n+1}\right) \subseteq C^{*}\left(H_{2 n+1}\right)$. Then

$$
\begin{aligned}
\pi_{\hbar}(F f) \phi(t) & =\frac{1}{(2 \pi)^{2 n+1}} \int(F f)(y, x, z) e^{i(\hbar z+y \cdot t)} \phi(t+\hbar x) d x d y d z \\
& =\frac{1}{(2 \pi)^{n}} \int f(t, \xi, \hbar) e^{-i\langle x, \xi\rangle} \phi(t+\hbar x) d \xi d x
\end{aligned}
$$

and, if we denote by $\hat{f}(t, x, \hbar)$ the function $\int f(t, \xi, \hbar) e^{-\imath\langle x, \xi\rangle} d \xi$, i.e., the Fourier transform of $f$ with respect to the second variable, then

$$
\pi_{\hbar}(F f) \phi(t)=\frac{1}{(2 \pi)^{n}} \int \hat{f}(t, x, \hbar) \phi(t+\hbar x) d x .
$$

Denote by $\rho_{\hbar}(\hat{f})$ the operator

$$
\rho_{\hbar}(\hat{f}) \phi(x)=\frac{1}{(2 \pi)^{n}} \int \hat{f}(x, y, \hbar) \phi(x+\hbar y) d y,
$$

which is a compact operator on $L^{2}\left(\mathbb{R}^{n}\right)$.

Define $\rho_{0}(\hat{f}) \in C_{0}\left(T^{*} \mathbb{R}\right)$ simply by $\rho_{0}(\hat{f})(x, \xi)=f(x, \xi, 0)$. Let $\rho(\hat{f})$ denote the vector field $\hbar \mapsto \rho_{\hbar}(\hat{f})$. The collection

$$
\Gamma=\left\{\rho(\hat{f}): f \in \mathscr{S}\left(T^{*} \mathbb{R}^{n} \times \mathbb{R}\right)\right\}
$$

verifies the assumptions of Proposition 10.2.3 of [D]. Hence, $\Gamma$ determines a unique structure of continuous field.

Remark 5.1. For any given $\mathscr{K}\left(L^{2}\left(\mathbb{R}^{n}\right)\right)$-valued continuous function $a$ on $(0,1]$, any $\varepsilon>0$, and any point $\hbar_{0} \in(0,1]$, there exists $f \in \mathscr{S}\left(T^{*} \mathbb{R}^{n} \times \mathbb{R}\right)$ such that $\left\|\rho_{\hbar}(\hat{f})-a(\hbar)\right\| \leqq \varepsilon$, for all $\hbar$ in some neighbourhood of $\hbar_{0}$. Thus, the field $\mathscr{A}^{\prime} \mid(0,1]$ induced by $\mathscr{A}^{\prime}$ on $(0,1]$ is a constant field. The field $\mathscr{A}^{\prime}$ is called a field trivial away from a point.

By [E-N-N, Theorem 2.4], the family $\mathscr{A}=(A(\hbar))$, where

$$
A(\hbar)= \begin{cases}C_{0}\left(T^{*} \mathbb{R}^{n} ; \operatorname{End}(V)\right), & \hbar=0, \\ \mathscr{K}\left(L^{2}\left(\mathbb{R}^{n} ; V\right)\right), & 0<\hbar \leqq 1,\end{cases}
$$

is in a natural way a continuous field, trivial away from 0 . Furthermore, $\mathscr{A}^{\sim}=$ $\left(A(\hbar)^{\sim}\right)$ is also a continuous field (see the proof of [E-N-N, Theorem 3.1]).

Let $P: \mathscr{S}\left(\mathbb{R}^{n} ; V\right) \rightarrow \mathscr{S}\left(\mathbb{R}^{n} ; V\right)$ be an elliptic operator with symbol $a$ of order $m>0$. For $\hbar>0$, let $P_{\hbar}$ denote the elliptic operator given by the symbol $a_{\hbar}(x, \xi)=$ $a(x, \hbar \xi)$. Denote by $e_{\hbar}$ the graph projection of $P_{\hbar}$. Set $e_{0}=e_{a}\left(e_{a}\right.$ is defined in Sect. 3).

The crucial technical result needed is given by the continuity property of the family of projections $e_{\hbar}$ stated below, and its proof will occupy us for the main part of this section.

Theorem 5.2. The vector field $e=\left(e_{\hbar}\right)$ of $M_{2}\left(\mathscr{A}^{\sim}\right)$ is continuous. 
Proof. For notational simplicity we restrict ourselves to the case of $\Psi$ DOs acting on scalar-valued functions. Before embarking on the proof, let us explain the strategy. The proof splits naturally into two cases.

In Part 1 we will prove the continuity at $\hbar>0$. This is the easy case, and it consists of showing that if the symbols are close in a well chosen topology, the corresponding operators are close in norm.

The interlude will contain certain more or less standard constructions and results on various kinds of symbols associated to pseudodifferential operators and their calculus.

In Part 2 we will prove the continuity at $\hbar=0$. Here the argument is more delicate. Given an elliptic symbol $a$ of order $m>0$, we have to show, for instance, the continuity of the field $\left(1+P_{\hbar}^{*} P_{\hbar}\right)^{-1}$. Since for each fixed $\hbar$ the operator $(1+$ $\left.P_{\hbar}^{*} P_{\hbar}\right)^{-1}$ is defined by functional calculus, the field $\left(1+P_{\hbar}^{*} P_{\hbar}\right)^{-1}$ is no more defined by rescaling of a suitable symbol. Also by construction, the value of this vector field at $\hbar=0$ is the operator of pointwise multiplication by the symbol $\left(1+a^{*} a\right)^{-1}$ of order $-2 m$, which, in turn, gives rise to the field of operators $\operatorname{Op}\left(\left(1+a^{*} a\right)_{\hbar}^{-1}\right)$ by rescaling the symbol $\left(1+a^{*} a\right)^{-1}$. Knowing that the latter is continuous (this is the content of Lemma 5.4), it is sufficient to approximate $\left(1+P_{\hbar}^{*} P_{\hbar}\right)^{-1}$ by $\mathrm{Op}((1+$ $\left.a^{*} a\right)_{\hbar}^{-1}$ ) around $\hbar=0$. This will be done in part two by analysing the behaviour of a symbol of $\left(1+P_{\hbar}^{*} P_{\hbar}\right)^{-1}$ as $\hbar \rightarrow 0$.

Part 1 Continuity at $\hbar_{0}>0$. In order to show the continuity of $e$, it is enough to show the continuity of $\hat{e}=e-\left(\begin{array}{ll}0 & 0 \\ 0 & 1\end{array}\right)$, because the vector field $\hbar \mapsto\left(\begin{array}{ll}0 & 0 \\ 0 & 1\end{array}\right)$ is continuous, by construction.

From now on throughout the proof of Theorem 5.2, by abuse of notation, for a given $\Psi$ DO $P$, let us denote by $P$ also the closure of $P \mid \mathscr{S}\left(\mathbb{R}^{n}\right)$.

Since $1+P_{\hbar_{0}}^{*} P_{\hbar_{0}}$ is of order $2 m>0$ and is invertible on $L^{2}\left(\mathbb{R}^{n}\right)$, by [Sh, Theorem 25.4] there exists a $\Psi$ DO $R_{0}$ of order $-2 m$ such that $\left(1+P_{\hbar_{0}}^{*} P_{\hbar_{0}}\right)^{-1}$ is the extension by continuity of $R_{0}$. The $\Psi \mathrm{DO}\left(1+P_{\hbar}^{*} P_{\hbar}\right) R_{0}$ is of order 0 , and hence is bounded on $L^{2}\left(\mathbb{R}^{n}\right)$. Consider the path of operators

$$
\begin{aligned}
(0,1] & \rightarrow B\left(L^{2},\left(\mathbb{R}^{n}\right)\right), \\
\hbar & \mapsto\left(1+P_{\hbar}^{*} P_{\hbar}\right) R_{0} .
\end{aligned}
$$

We assert that this path is norm continuous.

For any $s \in \mathbb{R}$, let $Q^{s}=Q^{s}\left(\mathbb{R}^{n}\right)$ denote the Sobolev $s$-space [Sh, Definition 25.3]. Any $\Psi \mathrm{DO}$ of order $m$ defines a continuous mapping:

$$
Q^{s} \rightarrow Q^{s-m}
$$

[Sh, Proposition 25.4]. We have

$$
\begin{aligned}
\left\|\left(1+P_{\hbar}^{*} P_{\hbar}\right) R_{0}-\left(1+P_{\hbar_{0}^{\prime}}^{*} P_{\hbar_{0}^{\prime}}\right) R_{0}\right\| & =\left\|\left(P_{\hbar}^{*} P_{\hbar}-P_{\hbar_{0}^{\prime}}^{*} P_{\hbar_{0}^{\prime}}\right) R_{0}\right\| \\
& \leqq\left\|P_{\hbar}^{*} P_{\hbar}-P_{\hbar^{\prime}}^{*} P_{\hbar^{\prime}}\right\|_{2 m}\left\|R_{0}\right\|_{-2 m},
\end{aligned}
$$

where $\|\cdot\|_{2 m}$ is the norm on operators of order $2 m$ considered as linear operators from $Q^{2 m}$ to $Q^{0}\left(=L^{2}\left(\mathbb{R}^{n}\right)\right)$, and $\|\cdot\|_{-2 m}$ is the norm on operators of order $-2 m$ considered as linear mappings from $Q^{0}$ to $Q^{2 m}$. Since as $\hbar \rightarrow \hbar^{\prime}$, 
$\sigma\left(P_{\hbar}^{*} P_{\hbar}\right) \rightarrow \sigma\left(P_{\hbar^{\prime}}^{*} P_{\hbar^{\prime}}\right)$ as symbols of order $2 m$, and as $\left(1+P_{\hbar_{0}}^{*} P_{\hbar_{0}}\right) R_{0}=1$, there exist $r_{0}>0$ and $\delta>0$ such that

$$
\left\|\left(1+P_{\hbar}^{*} P_{\hbar}\right) R_{0}-1\right\| \leqq r_{0}<1 \text { for }\left|\hbar-\hbar_{0}\right|<\delta .
$$

This implies that $\left(1+P_{\hbar}^{*} P_{\hbar}\right) R_{0}$ is invertible for $\left|\hbar-\hbar_{o}\right|<\delta$. Hence there exists a bounded operator $Q_{\hbar}$ such that

$$
\left(1+P_{\hbar}^{*} P_{\hbar}\right) R_{0} Q_{\hbar}=1 .
$$

The operator $Q_{\hbar}$ is given by

$$
Q_{\hbar}=1+\sum_{n=1}^{\infty}\left(1-\left(1+P_{\hbar}^{*} P_{\hbar}\right) R_{0}\right)^{n},
$$

and one sees that as $\hbar \rightarrow \hbar_{0}, Q_{\hbar}$ is uniformly bounded.

From $(*)$ it follows that $\left(1+P_{\hbar}^{*} P_{\hbar}\right)^{-1}=R_{0} Q_{\hbar}$. Recall [Sh, Proposition 25.4] that the canonical inclusion $Q^{2 m} \hookrightarrow Q^{0}$ is bounded. Thus, there exists a $C>0$ such that

$$
\|S\| \leqq C\|S\|_{-2 m}
$$

for every $\Psi$ DO $S$ of order $-2 m$. Hence,

$$
\left\|\left(1+P_{\hbar}^{*} P_{\hbar}\right)^{-1}\right\| \leqq C\left\|\left(1+P_{\hbar}^{*} P_{\hbar}\right)^{-1}\right\|_{-2 m} \leqq C\left\|R_{0}\right\|_{-2 m}\left\|Q_{\hbar}\right\| .
$$

Therefore, $\left(1+P_{\hbar}^{*} P_{\hbar}\right)^{-1}$ is uniformly bounded for $\left|\hbar-\hbar_{0}\right|<\delta$. We have

$$
\begin{aligned}
& \left\|\left(1+P_{\hbar}^{*} P_{\hbar}\right)^{-1}-\left(1+P_{\hbar_{0}}^{*} P_{\hbar_{0}}\right)^{-1}\right\| \\
& \quad \leqq C\left\|\left(1+P_{\hbar}^{*} P_{\hbar}\right)^{-1}-\left(1+P_{\hbar_{0}}^{*} P_{\hbar_{0}}\right)^{-1}\right\|_{-2 m} \\
& \quad \leqq C\left\|\left(1+P_{\hbar}^{*} P_{\hbar}\right)^{-1}\left(P_{\hbar_{0}}^{*} P_{\hbar_{0}}-P_{\hbar}^{*} P_{\hbar}\right)\left(1+P_{\hbar_{0}}^{*} P_{\hbar_{0}}\right)^{-1}\right\|_{-2 m} \\
& \quad \leqq C\left\|\left(1+P_{\hbar}^{*} P_{\hbar}\right)^{-1}\right\|_{-2 m}\left\|P_{\hbar_{0}}^{*} P_{\hbar_{0}}-P_{\hbar}^{*} P_{\hbar}\right\|_{2 m}\left\|\left(1+P_{\hbar_{0}}^{*} P_{\hbar_{0}}\right)^{-1}\right\|_{-2 m} \\
& \quad \leqq C\left\|R_{0}\right\|_{-2 m}\left\|Q_{\hbar}\right\|\left\|P_{\hbar_{0}}^{*} P_{\hbar_{0}}-P_{\hbar}^{*} P_{\hbar}\right\|_{2 m}\left\|R_{0}\right\|_{-2 m} .
\end{aligned}
$$

Consequently,

$$
\left\|\left(1+P_{\hbar}^{*} P_{\hbar}\right)^{-1}-\left(1+P_{\hbar_{0}}^{*} P_{\hbar_{0}}\right)^{-1}\right\| \rightarrow 0 \quad \text { as } \hbar \rightarrow \hbar_{0}
$$

Then

$$
\begin{aligned}
\| P_{\hbar}(1 & \left.+P_{\hbar}^{*} P_{\hbar}\right)^{-1}-P_{\hbar_{0}}\left(1+P_{\hbar_{0}}^{*} P_{\hbar_{0}}\right)^{-1} \|_{-m} \\
\leqq & \left\|\left(P_{\hbar}-P_{\hbar_{0}}\right)\left(1+P_{\hbar}^{*} P_{\hbar}\right)^{-1}\right\|_{-m} \\
& +\left\|P_{\hbar_{0}}\left(\left(1+P_{\hbar}^{*} P_{\hbar}\right)^{-1}-\left(1+P_{\hbar_{0}}^{*} P_{\hbar_{0}}\right)^{-1}\right)\right\|_{-m} \\
\leqq & \left\|P_{\hbar}-P_{\hbar_{0}}\right\|_{-m,-2 m}\left\|\left(1+P_{\hbar}^{*} P_{\hbar}\right)^{-1}\right\|_{-2 m} \\
& +\left\|P_{\hbar_{0}}\right\|_{-m,-2 m}\left\|\left(1+P_{\hbar}^{*} P_{\hbar}\right)^{-1}-\left(1+P_{\hbar_{0}}^{*} P_{\hbar_{0}}\right)^{-1}\right\|_{-2 m},
\end{aligned}
$$


where $\|\cdot\|_{-m,-2 m}$ is the norm on operators as linear transformations from $Q^{-2 m}$ to $Q^{-m}$. Therefore,

$$
\left\|P_{\hbar}\left(1+P_{\hbar}^{*} P_{\hbar}\right)^{-1}-P_{\hbar_{0}}\left(1+P_{\hbar_{0}}^{*} P_{\hbar_{0}}\right)^{-1}\right\|_{-m} \rightarrow 0 \quad \text { as } \hbar \rightarrow \hbar_{0} .
$$

Thus, the vector field $\hbar \mapsto P_{\hbar}\left(1+P_{\hbar}^{*} P_{\hbar}\right)^{-1}$ is continuous for $\hbar>0$.

Considering $P^{*}$ instead of $P$ one can show that both vector fields $\hbar \mapsto(1+$ $\left.P_{\hbar}^{*} P_{\hbar}\right)^{-1}$ and $\hbar \mapsto P_{\hbar}\left(1+P_{\hbar}^{*} P_{\hbar}\right)^{-1}$ are norm continuous for $\hbar>0$.

This completes the proof of Part 1.

Interlude. Before continuing with the proof, we will collect some more or less known facts on various kinds of symbols associated to $\Psi$ DOs on $\mathbb{R}^{n}$ and prove a few auxilary results about their behaviour under our assumptions. It should be perhaps pointed out that, in contrast to the case of $\mathbb{R}^{n}$, the results below are fairly standard in the case of closed manifolds.

Let $S^{k}$ denote the space of symbols of order $k$, considered in [Ku, Definition 1.1]; that is, $p \in C^{\infty}\left(T^{*} \mathbb{R}^{n}\right)$ belongs to $S^{k}$ if for any multi-indices $\alpha, \beta$, there exists a $C_{\alpha \beta}>0$ such that

$$
\left|D_{x}^{\alpha} D_{\xi}^{\beta} p(x, \xi)\right| \leqq C_{\alpha \beta}(1+|\xi|)^{k-|\beta|}, \quad(x, \xi) \in T^{*} \mathbb{R}^{n}
$$

The space $S^{k}$ is a Fréchet space with respect to the semi-norms

$$
|p|_{l}^{(k)}=\max _{|\alpha+\beta| \leqq l} \sup \left\{\left|D_{x}^{\alpha} D_{\xi}^{\beta} p(x, \xi)\right|(1+|\xi|)^{-k+|\beta|}:(x, \xi) \in T^{*} \mathbb{R}^{n}\right\} .
$$

Similarly, the space $\Gamma^{k}$ is topologized by the semi-norms

$$
\|\sigma\|_{l}^{(k)}=\max _{|\alpha+\beta| \leqq l} \sup \left\{\left|D_{x}^{\alpha} D_{\xi}^{\beta} \sigma(x, \xi)\right|(1+|x|+|\xi|)^{-k+|\alpha|+|\beta|}\right\} .
$$

Suppose that $k \leqq 0$. Let $\sigma \in \Gamma^{k}$. Then we have

$$
\left|D_{x}^{\alpha} D_{\xi}^{\beta} \sigma(x, \xi)\right| \leqq C_{\alpha \beta}(1+|x|+|\xi|)^{k-|\alpha|-|\beta|} \leqq C_{\alpha \beta}(1+|\xi|)^{k-|\beta|}
$$

This means that the space $\Gamma^{k}$ is contained in $S^{k}$, and the inclusion map $\Gamma^{k} \rightarrow S^{k}$ is continuous.

By Theorem 2.7 of $\left[\mathrm{Ku}\right.$, Chapter 2] there exist $C>0$ and $l_{0}$ such that for any $p \in S^{0}$, if we set $P=\operatorname{Op}(p)$, then

$$
\|P \phi\| \leqq C|p|_{l_{0}}^{(0)}\|\phi\|, \quad \phi \in \mathscr{S}\left(\mathbb{R}^{n}\right)
$$

That is,

$$
\|P\| \leqq C|p|_{l_{0}}^{(0)}
$$

Therefore, the map

$$
\begin{gathered}
S^{0} \rightarrow B\left(L^{2}\left(\mathbb{R}^{n}\right)\right) \\
p \mapsto P=\mathrm{Op}(p)
\end{gathered}
$$

is continuous. 
Lemma 5.4. Suppose that $\sigma \in \Gamma^{k}, k<0$, with $\|\sigma\|_{l_{0}}^{(k)}<\varepsilon$. Denote by $\sigma_{\hbar}$ the rescaled symbol $\sigma_{\hbar}(x, \xi)=\sigma(x, \hbar \xi)$. Then

$$
\left|\sigma_{\hbar}\right|_{l_{0}}^{(0)}<\varepsilon \text { for all } 0<\hbar \leqq 1 .
$$

Proof. Let $\alpha, \beta$ be multi-indices such that $|\alpha+\beta| \leqq l_{0}$. By definition,

$$
\left|D_{x}^{\alpha} D_{\xi}^{\beta} \sigma(x, \xi)\right| \leqq\|\sigma\|_{l_{0}}^{(k)}(1+|x|+|\xi|)^{k-|\alpha|-|\beta|} .
$$

We have

$$
\begin{aligned}
\left|D_{x}^{\alpha} D_{\xi}^{\beta} \sigma_{\hbar}(x, \xi)\right| & =\left|\hbar^{|\beta|}\left(D_{x}^{\alpha} D_{\xi}^{\beta} \sigma\right)(x, \hbar \xi)\right| \leqq \hbar^{|\beta|}\|\sigma\|_{l_{0}}^{(k)}(1+|x|+\hbar|\xi|)^{k-|\alpha|-|\beta|} \\
& \leqq\|\sigma\|_{l_{0}}^{(k)} \hbar^{|\beta|}(1+\hbar|\xi|)^{-|\beta|}=\|\sigma\|_{l_{0}}^{(k)}\left(\frac{1}{\hbar}+|\xi|\right)^{-|\beta|} \\
& \leqq\|\sigma\|_{l_{0}}^{(k)}(1+|\xi|)^{-|\beta|}
\end{aligned}
$$

as $\hbar \leqq 1$. Therefore,

$$
\left|\sigma_{\hbar}\right|_{l_{0}}^{(0)}=\max _{|\alpha+\beta| \leqq l_{0}} \sup \left|D_{x}^{\alpha} D_{\xi}^{\beta} \sigma_{\hbar}(x, \xi)\right|(1+|\xi|)^{|\beta|} \leqq\|\sigma\|_{l_{0}}^{(k)}<\varepsilon
$$

for all $0<\hbar \leqq 1$.

Given a symbol $\sigma \in \Gamma^{k}$, denote by $R(\sigma)$ the $\Psi$ DO with right symbol $\sigma$,

$$
R(\sigma) u(x)=\frac{1}{(2 \pi)^{n}} \int e^{i\langle x-y, \xi\rangle} \sigma(y, \xi) u(y) d y d \xi,
$$

while the $\Psi \mathrm{DO}$ defined by (2.3) is regarded as a $\Psi \mathrm{DO}$ with left symbol $\sigma$,

$$
L(\sigma) u(x)=\frac{1}{(2 \pi)^{n}} \int e^{i\langle x-y, \xi\rangle} \sigma(x, \xi) u(y) d y d \xi .
$$

An advantage of considering $\Psi$ DOs with right symbol is that the formal adjoint $L(\sigma)^{*}$ of $L(\sigma)$ is given by $R\left(\sigma^{*}\right)$. We need another useful notion from [Sh]. Recall that $b(x, y, \xi) \in C^{\infty}\left(\mathbb{R}^{3 n}\right)$ is an amplitude of order $k$ if for some $k^{\prime}$, we have

$$
\begin{aligned}
& \left|\partial_{\xi}^{\alpha} \partial_{x}^{\beta} \partial_{y}^{\gamma} b(x, y, \xi)\right| \\
& \quad \leqq C_{\alpha \beta \gamma}(1+|x|+|y|+|\xi|)^{k-|\alpha|-|\beta|-|\gamma|}(1+|x-y|)^{k^{\prime}+|\alpha|+|\beta|+|\gamma|} .
\end{aligned}
$$

For convenience we will say that such a $b$ is of order $k$ and of degree $k^{\prime}$, and denote by $\prod^{k, k^{\prime}}$ the space of amplitudes of order $k$ and of degree $k^{\prime}$. For $b \in \prod^{k, k^{\prime}}$, an operator $P(b)$ is defined by

$$
P(b) u(x)=\frac{1}{(2 \pi)^{n}} \int e^{i\langle x-y, \xi\rangle} b(x, y, \xi) u(y) d y d \xi .
$$

For $b \in \prod^{k, k^{\prime}}$, set $b_{\hbar}(x, y, \xi)=b(x, y, \hbar \xi)$. 
Lemma 5.6. Let $\sigma \in \Gamma^{m}$ and let $l$ be a positive integer such that $2 l-m$ is strictly positive. There exist $\sigma^{j} \in \Gamma^{m-2 j}, j=1, \ldots, l-1$, and $b \in \prod^{m-2 l, k^{\prime}}$ with $k^{\prime}>0$ such that

$$
L\left(\sigma_{\hbar}\right)=R\left(\sigma_{\hbar}\right)+\sum_{j=1}^{l-1} \hbar^{j} R\left(\sigma_{\hbar}^{j}\right)+\hbar^{l} \mathrm{Op}\left(b_{\hbar}\right) .
$$

Proof. The proof is modeled on that of [Sh, Theorem 23.2]. We present a complete proof. (It should perhaps be mentioned that the proof of [Sh, Theorem 23.2] is not correct as given; it uses an invalid inequality in Line 2 of page 174.)

Set $w=x-y$, so that

$$
\sigma_{\hbar}(x, \xi)=\sigma_{\hbar}(y+w, \xi)=\sigma(y+w, \hbar \xi) .
$$

Using Taylor's formula at $w=0$, we get

$$
\sigma(y+w, \hbar \xi)=\sigma(y, \hbar \xi)+\sum_{0<|\alpha|<l} \frac{1}{\alpha !} w^{\alpha}\left(\partial_{x}^{\alpha} \sigma\right)(y, \hbar \xi)+r_{l}(x, y, \xi),
$$

where

$$
r_{l}(x, y, \xi)=\sum_{|\alpha|=l} \frac{1}{\alpha !} w^{\alpha} \int_{0}^{1}(1-t)^{l-1}\left(\partial_{x}^{\alpha} \sigma\right)(y+t w, \hbar \xi) d t .
$$

The $\Psi$ DO with right symbol $(x-y)^{\alpha}\left(\partial_{x}^{\alpha} \sigma\right)(y, \hbar \xi)$ coincides with the $\Psi$ DO with right symbol

$$
\left(-D_{\xi}\right)^{\alpha}\left(\partial_{x}^{\alpha} \sigma(y, \hbar \xi)\right)=i^{|\alpha|} \hbar^{|\alpha|}\left(\partial_{\xi}^{\alpha} \partial_{x}^{\alpha} \sigma\right)(y, \hbar \xi),
$$

which is of order $m-2|\alpha|$. Set

$$
\sigma^{j}=\sum_{|\alpha|=j} \frac{i^{|\alpha|}}{\alpha !}\left(\partial_{\xi}^{\alpha} \partial_{x}^{\alpha} \sigma\right)
$$

The operator with amplitude $r_{l}$ is equal to that with amplitude

$$
\begin{aligned}
& \sum_{|\alpha|=l} \frac{1}{\alpha !} \int_{0}^{1}(1-t)^{l-1}\left(-D_{\xi}\right)^{\alpha}\left(\left(\partial_{x}^{\alpha} \sigma\right)(y+t w, \hbar \xi)\right) \\
& \quad=\sum_{|\alpha|=l} \hbar^{l} \cdot \frac{1}{\alpha !} \int_{0}^{1}(1-t)^{l-1}\left(\left(-D_{\xi}\right)^{\alpha} \partial_{x}^{\alpha} \sigma\right)(y+t w, \hbar \xi) d t .
\end{aligned}
$$

Let us show that this amplitude belongs to $\prod^{m-2 l, k^{\prime}}$ with $k^{\prime}>0$. It is enough to show that the integrand is estimated uniformly in $t$. Since $\sigma \in \Gamma^{m}$, one has

$$
\left|\left(\left(-D_{\xi}\right)^{\alpha} \partial_{x}^{\alpha} \sigma\right)(y+t w, \hbar \xi)\right| \leqq C_{\alpha}(1+|y+t w|+|\hbar \xi|)^{m-2 l}
$$

Choose $l$ such that $m-2 l<0$. Then

$$
(1+|y+t w|+|\hbar \xi|)^{m-2 l} \leqq(1+|w|+|y+t w|+|\hbar \xi|)^{m-2 l}(1+|w|)^{2 l-m} .
$$

It is easy to see that

$$
\frac{1}{2} \leqq \frac{|w|+|y+t w|}{|x|+|y|} \leqq 2
$$


for all $x, y \in \mathbb{R}^{n}, 0 \leqq t \leqq 1, w=x-y$. Hence,

$$
\left|\left(\left(-D_{\xi}\right)^{\alpha} \partial_{x}^{\alpha} \sigma\right)(y+t w, \hbar \xi)\right| \leqq C^{\prime}(1+|x|+|y|+|\hbar \xi|)^{m-2 l}(1+|x-y|)^{2 l-m},
$$

where $C^{\prime}$ is independent of $t$. In a similar fashion, one obtains estimates for derivatives. Therefore, if we set

$$
b(x, y, \xi)=\sum_{|\alpha|=e} \frac{1}{\alpha !} \int_{1}(1-t)^{l-1}\left(\left(-D_{\xi}\right)^{\alpha} \partial_{x}^{\alpha} \sigma\right)(y+t w, \xi) d t,
$$

then $b \in \prod^{m-2 l, 2 l-m}, 2 l-m>0$. Thus,

$$
L\left(\sigma_{\hbar}\right)=R\left(\sigma_{\hbar}\right)+\sum_{j=1}^{l-1} \hbar^{j} R\left(\sigma_{\hbar}^{j}\right)+\hbar^{l} \mathrm{Op}\left(b_{\hbar}\right),
$$

as required.

The next lemma gives a convenient way of constructing left symbols for operators defined by amplitudes from the class $\prod^{k, k^{\prime}}$.

Lemma 5.7. Suppose that $b \in \prod^{k, k^{\prime}}$ with $k \leqq 0, k^{\prime}>0$. Then the left symbol $\sigma(A)$ of $A=\mathrm{Op}(b)$ is given by

$$
\sigma(A)(x, \xi)=O_{s^{-}} \frac{1}{(2 \pi)^{n}} \int e^{-i y \cdot \eta} b(x, x+y, \xi+\eta) d y d \eta
$$

(oscillating integral, see e g., $[\mathrm{Ku}]$ ).

Proof. For $u \in \mathscr{S}\left(\mathbb{R}^{n}\right)$, one has

$$
\begin{aligned}
A u(x) & =\frac{1}{(2 \pi)^{n}} \int e^{i\langle x-y, \xi\rangle} b(x, y, \xi) u(y) d y d \xi \\
& =\frac{1}{(2 \pi)^{n}} \int e^{-i\langle y, \xi\rangle} b(x, x+y, \xi) u(x+y) d y d \xi
\end{aligned}
$$

As a function of $(y, \xi)$, the function $b(x, x+y, \xi) u(x+y)$ belongs to the class for which oscillating integrals are defined, see [Ku, Chapter 1]. In fact,

$$
\begin{aligned}
& \left|\left(\partial_{\xi}^{\alpha} \partial_{y}^{B}\right) b(x, x+y, \xi) u(x+y)\right| \\
& \quad \leqq \sum_{\beta_{1}+\beta_{2}=\beta}\left|\left(\partial_{\xi}^{\alpha} \partial_{y}^{\beta_{1}} b\right)(x, x+y, \xi) \partial_{y}^{\beta_{2}} u(x+y)\right| \\
& \quad \leqq \sum C_{\alpha \beta_{1}}(1+|x|+|y|+|\xi|)^{k-|\alpha|-\left|\beta_{1}\right|}(1+|y|)^{k^{\prime}+|\alpha|+\left|\beta_{1}\right|}\left|\partial_{y}^{\beta_{2}} u(x+y)\right| .
\end{aligned}
$$

Since $u \in \mathscr{S}\left(\mathbb{R}^{n}\right),\left|\partial_{y}^{\beta_{2}} u(x+y)\right|$ is bounded. Therefore

$$
\begin{aligned}
& \left|\left(\partial_{x}^{\alpha} \partial_{y}^{\beta}\right) b(x, x+y, \xi) u(x+y)\right| \\
& \quad \leqq \sum C_{\alpha \beta_{1}}^{\prime}(1+|\xi|)^{k}(1+|y|)^{-|\alpha|-\left|\beta_{1}\right|}(1+|y|)^{k^{\prime}+|\alpha|+\left|\beta_{1}\right|} \\
& \quad \leqq C(1+|\xi|)^{k}(1+|y|)^{k^{\prime}}
\end{aligned}
$$


Therefore,

$$
A u(x)=O_{s^{-}} \frac{1}{(2 \pi)^{n}} \int e^{-i\langle y, \xi\rangle} b(x, x+y, \xi) u(x+y) d y d \xi
$$

Notice that the last expression is valid for any function $u$ with all derivatives bounded, i.e., such that

$$
\left|\partial_{x}^{\alpha} u(x)\right| \leqq C_{\alpha} \quad \text { for any } \alpha
$$

By (23.57) of [Sh],

$$
\begin{aligned}
\sigma(A)(x, \xi) & =e^{-i x \cdot \xi} A\left(e^{i x \cdot \xi}\right) \\
& =O_{s^{-}} \frac{1}{(2 \pi)^{n}} \int e^{-i\langle y, \eta\rangle} b(x, x+y, \xi+\eta) d y d \eta .
\end{aligned}
$$

For later reference we record the following easy inequality.

Lemma 5.8. For $k>0$, one has

$$
(1+|x|+|\xi+\eta|)^{k} \leqq(1+|x|+|\xi|)^{k}(1+|\eta|)^{-k}
$$

Part 2. Continuity at $\hbar=0$. In order to compare $e_{0}$ and $e_{\hbar}(\hbar>0)$, we need to realize the $C^{*}$-algebras $A(\hbar)$ on a single Hilbert space.

For $\hbar>0$, consider the unitary $u_{\hbar}$ of $L^{2}\left(T^{*} \mathbb{R}^{n}\right)$ defined by

$$
\left(u_{\hbar} \phi\right)(x, \xi)=e^{\frac{l}{\hbar}\langle x, \xi\rangle} \phi(x, \xi), \quad \phi \in L^{2}\left(T^{*} \mathbb{R}^{n}\right) .
$$

Set $B(\hbar)=u_{\hbar}^{*}\left(\mathscr{K}\left(L^{2}\left(\mathbb{R}^{n}\right)\right) \otimes 1\right) u_{\hbar} \subseteq B\left(L^{2}\left(T^{*} \mathbb{R}^{n}\right)\right), \quad \hbar>0, \quad$ and $\quad B(0)=A(0)=$ $C_{0}\left(T^{*} \mathbb{R}^{n}\right)$.

By a straightforward computation,

$$
\begin{aligned}
\left(u_{\hbar}^{*}\left(\rho_{\hbar}(\hat{f}) \otimes 1\right) u_{\hbar}\right) \phi(x, \xi) & =\frac{1}{(2 \pi)^{n}} \int e^{i\langle y, \xi\rangle} \hat{f}(x, y, \hbar) \phi(x+\hbar y, \xi) d y \\
& =\frac{1}{(2 \pi)^{n}} \int e^{i\langle x-y, \eta\rangle} f(x, \xi+\hbar \eta, \hbar) \phi(y, \xi) d y d \eta
\end{aligned}
$$

for $f \in \mathscr{S}\left(T^{*} \mathbb{R}^{n} \times \mathbb{R}\right), \quad \phi \in L^{2}\left(T^{*} \mathbb{R}^{n}\right)$. For $f \in \mathscr{S}\left(T^{*} \mathbb{R}^{n} \times \mathbb{R}\right)$, define $\lambda_{\hbar}(f) \in$ $B\left(L^{2}\left(T^{*} \mathbb{R}^{n}\right)\right)$ by the right-hand side of $(* *)$. Notice that the right-hand side of $(* *)$ makes sense also for $\hbar=0$. In this case, the right-hand side of $(* *)$ is precisely the pointwise multiplication operator by the function $f(x, \xi, 0)$. So set $\lambda_{0}(f)$ equal to the pointwise multiplication operator by $f(\cdot, \cdot, 0)$. The function $\hbar \mapsto\left\|\lambda_{\hbar}(f)\right\|$ on $[0,1]$ is continuous (see, for instance, [R, Theorem 3.5]) and, the totality of vector fields $\lambda(f): \hbar \mapsto \lambda_{\hbar}(f)$ determines a unique structure of continuous field of $C^{*}$-algebras $\mathscr{B}=(B(\hbar))$ over $[0,1]$. It is clear that $\mathscr{A}$ and $\mathscr{B}$ are isomorphic.

Given a symbol $\sigma \in \Gamma^{k}$, let $P_{\sigma_{\hbar}}$ denote the $\Psi$ DO with symbol $\sigma_{\hbar}$. Set $\tilde{P}_{\sigma_{\hbar}}=$ $u_{\hbar}^{*}\left(P_{\sigma_{\hbar}} \otimes 1\right) u_{\hbar}, \hbar>0$. Then

$$
(* * *) \quad \tilde{P}_{\sigma_{\hbar}} \phi(x, \xi)=\frac{1}{(2 \pi)^{n}} \int e^{i\langle x-y, n\rangle} \sigma(x, \xi+\hbar \eta) \phi(y, \xi) d y d \eta
$$

for $\phi \in L^{2}\left(T^{*} \mathbb{R}^{n}\right)$. The right-hand side of $(* * *)$ makes sense when $\hbar=0$, and coincides with the pointwise multiplication operator by the function $\sigma \in C^{\infty}\left(T^{*} \mathbb{R}^{n}\right)$. 
Denote by $\tilde{P}_{\sigma}$ the field $\tilde{P}_{\sigma}=\left(\tilde{P}_{\sigma}(\hbar)\right)$ of operators

$$
\tilde{P}_{\sigma}(\hbar)= \begin{cases}\sigma, & \hbar=0, \\ \tilde{P}_{\sigma_{\hbar}}, & 0<\hbar \leqq 1 .\end{cases}
$$

By the definitions of the fields $\mathscr{B}$ and $\tilde{P}_{\sigma}$, we see that if $\sigma \in \Gamma^{-\infty}=\mathscr{S}\left(T^{*} \mathbb{R}^{n}\right)$, then $\tilde{P}_{\sigma}$ is a continuous section of $\mathscr{B}$. Actually, we have the following.

Lemma 5.9. If $\sigma \in \Gamma^{k}, k<0$, then $\tilde{P}_{\sigma}$ is a continuous section of $\mathscr{B}$.

Proof. By (5.3), for $\sigma \in S^{0}$, the norm $\left\|P_{\sigma}\right\|$ is estimated by $|\sigma|_{l_{0}}^{(0)}$. For a given $\varepsilon>0$, there exists $\sigma^{\prime} \in \Gamma^{-\infty}$ such that $\left\|\sigma-\sigma^{\prime}\right\|_{l_{0}}^{(k)}<\varepsilon$. Then by Lemma 5.4,

$$
\left|\sigma_{\hbar}-\sigma_{\hbar}^{\prime}\right|_{l_{0}}^{(0)}<\varepsilon \text { for all } 0<\hbar \leqq 1 \text {. }
$$

Hence for $\hbar>0$, we have

$$
\left\|\tilde{P}_{\sigma_{\hbar}}-\tilde{P}_{\sigma_{\hbar}^{\prime}}\right\|=\left\|P_{\sigma_{\hbar}}-P_{\sigma_{\hbar}^{\prime}}\right\|=\left\|P_{\sigma_{\hbar}-\sigma_{\hbar}^{\prime}}\right\| \leqq\left|\sigma_{\hbar}-\sigma_{\hbar}^{\prime}\right|_{l_{0}}^{(0)}<\varepsilon .
$$

It is clear that

$$
\left\|\tilde{P}_{\sigma}(0)-\tilde{P}_{\sigma^{\prime}}(0)\right\|=\sup \left|\sigma(x, \xi)-\sigma^{\prime}(x, \xi)\right| \leqq\left\|\sigma-\sigma^{\prime}\right\|_{l_{0}}^{(k)}<\varepsilon .
$$

The observation above means that $\tilde{P}_{\sigma}$ can be approximated by fields of the form $\tilde{P}_{\sigma^{\prime}}, \sigma^{\prime} \in \Gamma^{-\infty}$. Hence $\tilde{P}_{\sigma}$ itself is a continuous field.

Let now $a$ be elliptic of order $m>0$. We have to show the continuity of the following fields of operators:

$$
\begin{aligned}
& \left(1+\tilde{P}_{a}^{*} \tilde{P}_{a}\right)^{-1}, \\
& \left(1+\tilde{P}_{a}^{*} \tilde{P}_{a}\right)^{-1} \tilde{P}_{a}^{*}, \\
& \left(1+\tilde{P}_{a} \tilde{P}_{a}^{*}\right)^{-1} \text { and } \\
& \left(1+\tilde{P}_{a} \tilde{P}_{a}^{*}\right)^{-1} \tilde{P}_{a} .
\end{aligned}
$$

Before giving the proof, let us recall the strategy. Take, for instance, $(1+$ $\left.\tilde{P}_{a} \tilde{P}_{a}^{*}\right)^{-1}$. Since $a$ is elliptic of order $m>0$, the symbol $b$ defined by

$$
b(x, \xi)=\left(1+a(x, \xi) a(x, \xi)^{*}\right)^{-1}
$$

is of order $-2 m$. By construction,

$$
\tilde{P}_{b}(0)=\left(1+\tilde{P}_{a}(0) \tilde{P}_{a}(0)^{*}\right)^{-1}=\text { the pointwise multiplication by } b \text {. }
$$

By Part 1 , the field $\left(1+\tilde{P}_{a} \tilde{P}_{a}^{*}\right)^{-1}$ is continuous on $(0,1]$. By Lemma 5.9 , the field $\tilde{P}_{b}$ is continuous on $[0,1]$. Therefore, in order to show the continuity at $\hbar=0$, it is sufficient to verify that

$$
\left\|\left(1+\tilde{P}_{a}(\hbar) \tilde{P}_{a}(\hbar)^{*}\right)^{-1}-\tilde{P}_{b}(\hbar)\right\| \rightarrow 0 \quad \text { as } \hbar \rightarrow 0 .
$$

Notice that for $\hbar>0$, one has the equality

$$
\left\|\left(1+\tilde{P}_{a}(\hbar) \tilde{P}_{a}(\hbar)^{*}\right)^{-1}-\tilde{P}_{b}(\hbar)\right\|=\left\|\left(1+P_{a_{\hbar}} P_{a_{\hbar}}^{*}\right)^{-1}-P_{b_{\hbar}}\right\| .
$$

Thus we only have to show that

$$
\left\|\left(1+P_{a_{\hbar}} P_{a_{\hbar}}^{*}\right)^{-1}-P_{b_{\hbar}}\right\| \rightarrow 0 \quad \text { as } \hbar \rightarrow 0 .
$$

The main component of the proof is the following proposition. 
Proposition 5.10. Fix an elliptic $a \in \Gamma^{m}, m>0$ and set $P_{\hbar}=L\left(a_{\hbar}\right)$. The following holds:

$$
P_{\hbar} P_{\hbar}^{*}=R\left(a_{\hbar} a_{\hbar}^{*}\right)+\sum_{j=1}^{N-1} \hbar^{j} R\left(\sigma_{\hbar}^{j}\right)+\hbar^{N} \mathrm{Op}\left(b_{\hbar}\right),
$$

where $\sigma^{j} \in \Gamma^{2 m-2 j}$, and $b$ is an amplitude of order $2 m-2 N \leqq 0$ and of degree $4 N>0$. The operators $\mathrm{Op}\left(b_{\hbar}\right)$ are bounded uniformly in $\hbar$.

Proof. We can write:

$$
P_{\hbar}^{*} u(x)=R\left(a_{\hbar}^{*}\right) u(x)=\frac{1}{(2 \pi)^{n}} \int e^{i\langle x-y, \xi\rangle} a(y, \hbar \xi)^{*} u(y) d y d \xi,
$$

and

$$
\left(P_{\hbar} P_{\hbar}^{*}\right) u(x)=\frac{1}{(2 \pi)^{n}} \int e^{i\langle x-y, \xi\rangle} p(x, y, \hbar \xi) u(y) d y d \xi=\mathrm{Op}\left(p_{\hbar}\right),
$$

where $p(x, y, \xi)=a(x, \xi) a(y, \xi)^{*}$ is an amplitude of order $2 m$.

Set $w=x-y$, so that

$$
p(x, y, \hbar \xi)=p(y+w, y, \hbar \xi) .
$$

As in the proof of Lemma 5.6, use Taylor's formula at $w=0$ to get that

$$
p(y+w, y, \hbar \xi)=a(y, \hbar \xi) a(y, \hbar \xi)^{*}+\sum_{0<|\alpha|<N} \frac{1}{\alpha !} w^{\alpha}\left(\partial_{x}^{\alpha} p\right)(y, y, \hbar \xi)+r_{N}(x, y, \xi),
$$

where

$$
r_{N}(x, y, \xi)=\sum_{|\alpha|=N} \frac{1}{\alpha !} w^{\alpha} \int_{0}^{1}(1-t)^{N-1}\left(\partial_{x}^{\alpha} p\right)(y+t w, y, \hbar \xi) d t .
$$

The operator with amplitude

$$
(x-y)^{\alpha}\left(\partial_{x}^{\alpha} p\right)(y, y, \hbar \xi)
$$

is the same as the operator with symbol of order $2 m-2|\alpha|$,

$$
\left(-D_{\xi}^{\alpha}\right)\left(\partial_{x}^{\alpha} p(y, y, \hbar \xi)\right)=\left.\hbar^{|\alpha|} \partial_{x}^{\alpha}\left(-D_{\xi}^{\alpha}\right) p(x, y, \hbar \xi)\right|_{x=y} .
$$

Set

$$
\sigma_{j}(y, \xi)=\left.\sum_{|\alpha|=j} \frac{1}{\alpha !}\left(\partial_{x}^{\alpha}\left(-D_{\xi}\right)^{\alpha} p(x, y, \hbar \xi)\right)\right|_{x=y} .
$$

Then $\sigma_{J} \in \Gamma^{2 m-2 j}$.

The operator $\operatorname{Op}\left(\left(r_{N}\right)_{\hbar}\right)$ is equal to the operator with amplitude

$$
\begin{aligned}
& \sum_{|\alpha|=N} \frac{1}{\alpha !} \int_{0}^{1}\left(-D_{\xi}\right)^{\alpha}\left(\partial_{x}^{\alpha} p(y+t w, y, \hbar \xi)\right)(1-t)^{N-1} d t \\
& \quad=\sum_{|\alpha|=N} \frac{\hbar^{N}}{\alpha !} \int_{0}^{1}\left(\left(-D_{\xi}\right)^{\alpha} \partial_{x}^{\alpha} p\right)(y+t w, y, \hbar \xi)(1-t)^{N-1} d t .
\end{aligned}
$$


Set

$$
S_{N}(x, y, \xi)=\sum_{|\alpha|=N} \frac{1}{\alpha !} \int_{0}^{1}\left(\left(-D_{\xi}\right)^{\alpha} \partial_{x}^{\alpha} p\right)(y+t w, y, \hbar \xi)(1-t)^{N-1} d t .
$$

Assertion

$$
S_{N} \in \prod^{2 m-2 N, 4 N}
$$

First, observe that

$$
\left(\left(-D_{\xi}\right)^{\alpha} \partial_{x}^{\alpha} p\right)(y+t w, y, \xi)=\sum_{\alpha_{1}+\alpha_{2}=\alpha}\left(\left(-D_{\xi}\right)^{\alpha_{1}} \partial_{x}^{\alpha} a\right)(y+t w, \xi)\left(-D_{\xi}\right)^{\alpha_{2}} a(y, \xi)^{*}
$$

Now, we have

$$
\begin{aligned}
& \left|\left(-D_{\xi}\right)^{\alpha} \partial_{x}^{\alpha} p(x, y, \xi)\right| \\
& \leqq \sum_{\alpha_{1}+\alpha_{2}=\alpha} C_{\alpha_{1}}(1+|x|+|\xi|)^{m-|\alpha|-\left|\alpha_{1}\right|}(1+|y|+|\xi|)^{m-\left|\alpha_{2}\right|} \\
& \leqq \sum_{\alpha_{1}+\alpha_{2}=\alpha} C_{\alpha_{1}}(1+|x|+|x-y|+|\xi|)^{m-|\alpha|-\left|\alpha_{1}\right|}(1+|x-y|)^{|m-| \alpha_{1}|-| \alpha_{1}||} \\
& \times(1+|y|+|x-y|+|\xi|)^{m-\left|\alpha_{2}\right|}(1+|x-y|)^{|m-| \alpha_{2}||} \\
& \leqq \sum C_{\alpha_{1}}^{\prime}(1+|x|+|y|+|\xi|)^{2 m-|\alpha|-\left|\alpha_{1}\right|-\left|\alpha_{2}\right|} \\
& \times(1+|x-y|)^{|m-| \alpha|-| \alpha_{1}||+|m-| \alpha_{2}||} .
\end{aligned}
$$

Since $m>0$, we have

$$
|m-| \alpha|-| \alpha_{1}|| \leqq m+|\alpha|+\left|\alpha_{1}\right| \text { and }|m-| \alpha_{2}|| \leqq m+\left|\alpha_{2}\right| .
$$

Therefore,

$$
\left|\left(-D_{\xi}\right)^{\alpha} \partial_{x}^{\alpha} p(x, y, \xi)\right| \leqq C_{\alpha}(1+|x|+|y|+|\xi|)^{2 m-2 N}(1+|x-y|)^{2 m+2 N} .
$$

From this it follows that

$$
\begin{aligned}
& \left|\left(-D_{\xi}\right)^{\alpha} \partial_{x}^{\alpha} p(y+t w, y, \xi)\right| \\
& \quad \leqq C_{\alpha}(1+|y+t w|+|y|+|\xi|)^{2 m-2 N}(1+|t w|)^{2 m+2 N} \\
& \quad \leqq C_{\alpha}^{\prime}(1+|y|+|t w|+|\xi|)^{2 m-2 N}(1+|t w|)^{2 m+2 N} .
\end{aligned}
$$

Choose $N$ such that $2 m-2 N \leqq 0$. Then

$$
\begin{aligned}
(1+|y|+|t w|+|\xi|)^{2 m-2 N} & \leqq(1+|y|+|\xi|)^{2 m-2 N}, \\
(1+|t w|)^{2 m+2 N} & \leqq(1+|w|)^{2 m+2 N},
\end{aligned}
$$

the latter inequality following from the fact that $0 \leqq t \leqq 1,2 m+2 N>0$. Therefore,

$$
\begin{aligned}
\mid( & \left.-D_{\xi}\right)^{\alpha} \partial_{x}^{\alpha} p(y+t w, y, \xi) \mid \\
& \leqq C_{\alpha}^{\prime}(1+|y|+|\xi|)^{2 m-2 N}(1+|w|)^{2 m+2 N} \\
& \leqq C_{\alpha}^{\prime \prime}(1+|y|+|w|+|\xi|)^{2 m-2 N} \cdot(1+|w|)^{2 N-2 m} \cdot(1+|w|)^{2 m+2 N} \\
& \leqq C_{\alpha}^{\prime \prime \prime}(1+|x|+|y|+|\xi|)^{2 m-2 N} \cdot(1+|x-y|)^{4 N},
\end{aligned}
$$


and $C_{\alpha}^{\prime \prime \prime}$ is independent of $t$. Hence,

$$
\left|S_{N}(x, y, \xi)\right| \leqq C_{0}(1+|x|+|y|+|\xi|)^{2 m-2 N}(1+|x-y|)^{4 N} .
$$

In an analogous way one obtains estimates for higher derivatives of $S_{N}$. This proves that $S_{N}$ does indeed belong to $\prod^{2 m-2 N, 4 N}$.

We are now in position to apply Lemma 5.7, where we set $b=S_{N}$ and $k^{\prime}=4 N$. Set $A_{\hbar}=\operatorname{Op}\left(b_{\hbar}\right)$ and let $s, t$ be even non-negative integers. We consider the function of $(y, \eta)$,

$$
\langle\eta\rangle^{-s}\left\langle D_{y}\right\rangle^{s}\langle y\rangle^{-t}\left\langle D_{\eta}\right\rangle^{t} b(x, x+y, \hbar \xi+\hbar \eta)
$$

where $x$ and $\xi$ are regarded as parameters (we borrow the notation from [Sh]). By (5.5) and Lemma 5.8,

$$
\begin{aligned}
& \left|\langle\eta\rangle^{-s}\left\langle D_{y}\right\rangle^{s}\langle y\rangle^{-t}\left\langle D_{\eta}\right\rangle^{t} b(x, x+y, \hbar \xi+\hbar \eta)\right| \leqq C \sum_{2 \leqq p_{1}+p_{2} \leqq s} \sum_{j=0}^{t / 2}\left\{\langle\eta\rangle^{-s}\langle y\rangle^{-t-p_{1}}\right. \\
& \left.\quad \times(1+|x|+|y|+|\hbar \xi+\hbar \eta|)^{k-2 j-p_{2}} \times\langle y\rangle^{k^{\prime}+2 j+p_{2}} \hbar^{2 j}\right\} \\
& \quad \leqq C \sum_{j}\left\{\langle\eta\rangle^{-s}\langle y\rangle^{-t-p_{1}}\langle y\rangle^{k^{\prime}+2 j+p_{2}}\langle y\rangle^{-2 j-p_{2}} \times(1+|x|+|\hbar \xi+\hbar \eta|)^{k} \hbar^{2 j}\right\} \\
& \quad \leqq C \sum \sum_{j}\langle\eta\rangle^{-s}\langle y\rangle^{-t-p_{1}+k^{\prime}}(1+|x|+|\hbar \xi|)^{k}(1+|\hbar \eta|)^{-k} \\
& \leqq C^{\prime}\langle\eta\rangle^{-s-k}\langle y\rangle^{-t-k^{\prime}}(1+|x|+|\hbar \xi|)^{k} .
\end{aligned}
$$

Therefore, if one chooses $s, t$ such that $-s-k<-n$ and $-t+k^{\prime}<-n$, then the function

$$
\langle\eta\rangle^{-s}\left\langle D_{y}\right\rangle^{s}\langle y\rangle^{-t}\left\langle D_{\eta}\right\rangle^{t} b(x, x+y, \hbar \xi+\hbar \eta)
$$

is integrable as a function of $(y, \eta)$.

By Lemma 5.7,

$$
\begin{aligned}
\sigma\left(A_{\hbar}\right)(x, \xi) & =O_{s^{-}} \frac{1}{(2 \pi)^{n}} \int e^{-i\langle y, \eta\rangle} b(x, x+y, \hbar \xi+\hbar \eta) d y d \eta \\
& =\frac{1}{(2 \pi)^{n}} \int e^{-i\langle y, \eta\rangle}\langle\eta\rangle^{-s}\left\langle D_{y}\right\rangle^{s}\langle y\rangle^{-t}\left\langle D_{\eta}\right\rangle^{t} b(x, x+y, \hbar \xi+\hbar \eta) d y d \eta
\end{aligned}
$$

and

$$
\left|\sigma\left(A_{\hbar}\right)(x, \xi)\right| \leqq C_{0}(1+|x|+|\hbar \xi|)^{k}
$$

with $C_{0}$ independent of $\hbar$. By a similar computation,

$$
\left|\partial_{x}^{\alpha} \partial_{\xi}^{\beta} \sigma\left(A_{\hbar}\right)(x, \xi)\right| \leqq C_{\alpha \beta} \hbar^{|\beta|}(1+|x|+|\hbar \xi|)^{k-|\alpha|-|\beta|}
$$

with $C_{\alpha \beta}$ independent of $\hbar$. It then follows that $\left\{\sigma\left(A_{\hbar}\right)\right\}_{0<\hbar \leqq 1}$ is a bounded set in $S^{0}$. Now an application of (5.3) completes the proof of Proposition 5.10.

It should be pointed out that, in the case of $k=2 m-2 N$ strictly negative, although for each fixed $0<\hbar \leqq 1$, the symbol $\sigma\left(A_{\hbar}\right)$ is of order $k$, the set $\left\{\sigma\left(A_{\hbar}\right)\right\}$ is not bounded in the space $\Gamma^{\bar{k}}$, nor in $S^{k}$. 
We can now complete the proof of the second part of Theorem 5.2. By Proposition 5.10 we have the decomposition:

Now,

$$
1+P_{\hbar} P_{\hbar}^{*}=R\left(1+a_{\hbar} a_{\hbar}^{*}\right)+\sum_{j=1}^{N-1} \hbar^{j} R\left(\sigma_{\hbar}^{j}\right)+\hbar^{N} \mathrm{Op}\left(b_{\hbar}\right) .
$$

$$
\begin{aligned}
& L\left(\left(1+a_{\hbar} a_{\hbar}^{*}\right)^{-1}\right)\left(1+P_{\hbar} P_{\hbar}^{*}\right)=L\left(\left(1+a_{\hbar} a_{\hbar}^{*}\right)^{-1}\right) R\left(1+a_{\hbar} a_{\hbar}^{*}\right) \\
& \quad+\sum_{j=1}^{N-1} \hbar^{j} L\left(\left(1+a_{\hbar} a_{\hbar}^{*}\right)^{-1}\right) R\left(\sigma_{\hbar}^{j}\right)+\hbar^{N} L\left(\left(1+a_{\hbar} a_{\hbar}^{*}\right)^{-1}\right) \operatorname{Op}\left(b_{\hbar}\right) .
\end{aligned}
$$

By (5.10),

$$
\sup \left\{\left\|\mathrm{Op}\left(b_{\hbar}\right)\right\|: 0<\hbar \leqq 1\right\}<\infty
$$

and by (5.9),

$$
\sup \left\{\left\|L\left(\left(1+a_{\hbar} a_{\hbar}^{*}\right)^{-1}\right)\right\|: 0<\hbar \leqq 1\right\}<\infty .
$$

Therefore,

$$
\left\|\hbar^{N} L\left(\left(1+a_{\hbar} a_{\hbar}^{*}\right)^{-1}\right) \mathrm{Op}\left(b_{\hbar}\right)\right\| \rightarrow 0
$$

as $\hbar \rightarrow 0$.

If we set

$$
p(x, y, \xi)=\left(1+a(x, \xi) a(x, \xi)^{*}\right)^{-1} \sigma^{j}(y, \xi),
$$

then $p \in \prod^{-2 j, k^{\prime}}$ with $k^{\prime}>0$, and

$$
L\left(\left(1+a_{\hbar} a_{\hbar}^{*}\right)^{-1}\right) R\left(\sigma_{\hbar}^{j}\right)=\operatorname{Op}\left(P_{\hbar}\right) .
$$

Therefore,

$$
\left\|\sum_{j=1}^{N-1} \hbar^{j} L\left(\left(1+a_{\hbar} a_{\hbar}^{*}\right)^{-1}\right) R\left(\sigma_{\hbar}^{j}\right)\right\| \rightarrow 0
$$

as $\hbar \rightarrow 0$. It is easy to verify that

$$
L\left(\left(1+a_{\hbar} a_{\hbar}^{*}\right)^{-1}\right) R\left(1+a_{\hbar} a_{\hbar}^{*}\right)=L\left(\left(1+a_{\hbar} a_{\hbar}^{*}\right)^{-1}\left(1+a_{\hbar} a_{\hbar}^{*}\right)\right)+\hbar \mathrm{Op}\left(q_{\hbar}\right),
$$

where $q$ is an amplitude of order -2 and of degree $>0$. By (5.10),

$$
\left\|\hbar \mathrm{Op}\left(b_{\hbar}\right)\right\| \rightarrow 0 \quad \text { as } \hbar \rightarrow 0 \text {. }
$$

Obviously, $L\left(\left(1+a_{\hbar} a_{\hbar}^{*}\right)^{-1}\left(1+a_{\hbar} a_{\hbar}^{*}\right)\right)=1$. Therefore,

$$
\begin{aligned}
\left\|L\left(\left(1+a_{\hbar} a_{\hbar}^{*}\right)^{-1}\right)\left(1+P_{\hbar} P_{\hbar}^{*}\right)-1\right\| \leqq & \hbar\left\|\mathrm{Op}\left(q_{\hbar}\right)\right\|+\sum_{j=1}^{N-1} \hbar^{j} \| L\left(\left(1+a_{\hbar} a_{\hbar}^{*}\right)^{-1}\|\| R\left(\sigma_{\hbar}^{j}\right) \|\right. \\
& +\hbar^{N}\left\|L\left(\left(1+a_{\hbar} a_{\hbar}^{*}\right)^{-1}\right)\right\|\left\|\mathrm{Op}\left(b_{\hbar}\right)\right\|,
\end{aligned}
$$

and

$$
\left\|L\left(\left(1+a_{\hbar} a_{\hbar}^{*}\right)^{-1}\right)\left(1+P_{\hbar} P_{\hbar}^{*}\right)-1\right\| \rightarrow 0
$$

as $\hbar \rightarrow 0$.

For notational simplicity, set $R_{\hbar}=L\left(\left(1+a_{\hbar} a_{\hbar}^{*}\right)^{-1}\right)$. By $(5.11)$, there exist constants $\delta>0$ and $c>0$ such that

$$
\left\|1-R_{\hbar}\left(1+P_{\hbar} P_{\hbar}^{*}\right)\right\| \leqq C<1 \text { for } 0<\hbar \leqq \delta .
$$


This, in particular, means that if $0<\hbar \leqq \delta$, then $R_{\hbar}\left(1+P_{\hbar} P_{\hbar}^{*}\right)$ is invertible. Thus, there exists a bounded operator $S_{\hbar}$ such that

$$
S_{\hbar} R_{\hbar}\left(1+P_{\hbar} P_{\hbar}^{*}\right)=1,
$$

and this $S_{\hbar}$ is given by

$$
S_{\hbar}=1+\sum_{n=1}^{\infty}\left(1-R_{\hbar}\left(1+P_{\hbar} P_{\hbar}^{*}\right)\right)^{n} .
$$

We know that $\left\|S_{\hbar}-1\right\| \rightarrow 0$ as $\hbar \rightarrow 0$. We have that $\left(1+P_{\hbar} P_{\hbar}^{*}\right)^{-1}=S_{\hbar} R_{\hbar}$ and that

$$
\left\|\left(1+P_{\hbar} P_{\hbar}^{*}\right)^{-1}\right\| \leqq\left\|S_{\hbar}\right\|\left\|R_{\hbar}\right\| \text {. }
$$

From this it follows that

$$
\sup \left\{\left\|\left(1+P_{\hbar} P_{\hbar}^{*}\right)^{-1}\right\|: 0<\hbar \leqq \delta\right\}<\infty .
$$

We have the inequality

$$
\begin{aligned}
\|\left(1+P_{\hbar} P_{\hbar}^{*}\right)^{-1}-L\left(\left(1+a_{\hbar} a_{\hbar}^{*}\right)^{-1} \|\right. & =\left\|\left\{1-R_{\hbar}\left(1+P_{\hbar} P_{\hbar}^{*}\right)\right\}\left(1+P_{\hbar} P_{\hbar}^{*}\right)^{-1}\right\| \\
& \leqq\left\|1-R_{\hbar}\left(1+P_{\hbar} P_{\hbar}^{*}\right)\right\|\left\|\left(1+P_{\hbar} P_{\hbar}^{*}\right)^{-1}\right\| .
\end{aligned}
$$

Therefore,

$$
\|\left(1+P_{\hbar} P_{\hbar}^{*}\right)^{-1}-L\left(\left(1+a_{\hbar} a_{\hbar}^{*}\right)^{-1} \| \rightarrow 0\right.
$$

as $\hbar \rightarrow 0$. We already know that $\left(1+P_{\hbar} P_{\hbar}^{*}\right)^{-1}$ is continuous for $\hbar>0$. Hence by the property (iv) of Definition 10.1.2 of [D], $\left(1+P_{\hbar} P_{\hbar}^{*}\right)^{-1}$ is continuous at $\hbar=0$.

Next, we turn to the proof of the continuity of $\left(1+P_{\hbar} P_{\hbar}^{*}\right)^{-1} P_{\hbar}$ at $\hbar=0$. The strategy is the same as above. So, let us briefly discuss the proof.

By construction, $\left(1+\tilde{P}_{a}(0) \tilde{P}_{a}(0)^{*}\right)^{-1} \tilde{P}_{a}(0)$ is the pointwise multiplication operator by the function $\left(1+a a^{*}\right)^{-1} a$. Therefore, it is enough to verify that

$$
\left\|\left(1+P_{\hbar} P_{\hbar}^{*}\right)^{-1} P_{\hbar}-L\left(\left(1+a_{\hbar} a_{\hbar}^{*}\right)^{-1} a_{\hbar}\right)\right\| \rightarrow 0
$$

as $\hbar \rightarrow 0$.

It is not difficult to see that for $0<\hbar \leqq \delta$, the operator $\left(1+P_{\hbar} P_{\hbar}^{*}\right)^{-1} P_{\hbar}$ is written in the form

$$
\left(1+P_{\hbar} P_{\hbar}^{*}\right)^{-1} P_{\hbar}=S_{\hbar}\left\{L\left(\left(1+a_{\hbar} a_{\hbar}^{*}\right)^{-1} a_{\hbar}\right)+\hbar \mathrm{Op}\left(P_{\hbar}\right)\right\},
$$

where $p$ is an amplitude of negative order and of positive degree. It follows then that

$$
\left(1+P_{\hbar} P_{\hbar}^{*}\right)^{-1}-L\left(\left(1+a_{\hbar} a_{\hbar}^{*}\right)^{-1} a_{\hbar}\right)=\left(S_{\hbar}-1\right) L\left(\left(1+a_{\hbar} a_{\hbar}^{*}\right)^{-1} a_{\hbar}\right)+\hbar S_{\hbar} \mathrm{Op}\left(p_{\hbar}\right),
$$

and that

$$
\left\|\left(1+P_{\hbar} P_{\hbar}^{*}\right)^{-1} P_{\hbar}-L\left(\left(1+a_{\hbar} a_{\hbar}^{*}\right)^{-1} a_{\hbar}\right)\right\| \rightarrow 0
$$

as $\hbar \rightarrow 0$.

Thus the continuity of $\left(1+P_{\hbar} P_{\hbar}^{*}\right)^{-1} P_{\hbar}$ at $\hbar=0$ is proved.

One can show the continuity of $\left(1+P_{\hbar} P_{\hbar}^{*}\right)^{-1} P_{\hbar}$, and $\left(1+P_{\hbar}^{*} P_{\hbar}\right)^{-1}$ in a similar, though perhaps more tedious manner.

This completes the proof of Theorem 5.2. 
Proof of Theorem 3.1. Once the continuity of the field of graph projections is established, we can prove the index theorem, Theorem 3.1, by generalizing the arguments of [E-N-N, 4.7]. However, in the present paper we give a more visual presentation of the idea behind the proof, which will occupy us for the rest of this section.

For an elliptic operator $P: \mathscr{S}\left(\mathbb{R}^{n} ; V\right) \rightarrow \mathscr{S}\left(\mathbb{R}^{n} ; V\right)$, the graph projection $e$ belongs to $M_{2}\left(\mathscr{K}\left(L^{2}\left(\mathbb{R}^{n} ; V\right)\right)^{\sim}\right)$, as we know. If $\operatorname{dim} V=d$, then

$$
\begin{aligned}
\mathscr{K}\left(L^{2}\left(\mathbb{R}^{n} ; V\right)\right) & =M_{d}\left(\mathscr{K}\left(L^{2}\left(\mathbb{R}^{n}\right)\right)\right), \\
\mathscr{K}\left(L^{2}\left(\mathbb{R}^{n} ; V\right)\right)^{\sim} & =M_{d}\left(\mathscr{K}\left(L^{2}\left(\mathbb{R}^{n}\right)\right)\right)^{\sim} \subseteq M_{d}\left(\mathscr{K}\left(L^{2}\left(\mathbb{R}^{n}\right)\right)^{\sim}\right) .
\end{aligned}
$$

Thus,

$$
e \in M_{2 d}\left(\mathscr{K}\left(L^{2}\left(\mathbb{R}^{n}\right)\right)^{\sim}\right) \quad \text { and } \quad \hat{e}=e-\left(\begin{array}{ll}
0 & 0 \\
0 & 1
\end{array}\right) \in M_{2 d}\left(\mathscr{K}\left(L^{2}\left(\mathbb{R}^{n}\right)\right)\right),
$$

where $\left(\begin{array}{ll}0 & 0 \\ 0 & I\end{array}\right) \in M_{2 d}(\mathbb{C})$, and $I$ is the $d \times d$ identity matrix.

For each $\hbar>0$, the sub-*-algebra $\left\{\rho_{\hbar}(\hat{f}) ; t \in \mathscr{S}\left(T^{*} \mathbb{R}^{n} \times \mathbb{R}\right)\right\}$ is precisely the sub-algebra $\mathscr{K}^{\infty}$ of integral operators with integral kernels in $\mathscr{S}\left(\mathbb{R}^{n} \times \mathbb{R}^{n}\right)$, and hence is closed under the holomorphic functional calculus in $\mathscr{K}\left(L^{2}\left(\mathbb{R}^{n}\right)\right)$. As for the case of $\hbar=0$, the sub-*-algebra $\left\{\rho_{0}(\hat{f}) ; f \in \mathscr{S}\left(T^{*} \mathbb{R}^{n} \times \mathbb{R}\right)\right\}$ is the subalgebra $\mathscr{S}\left(T^{*} \mathbb{R}^{n}\right)$ of $C_{0}\left(T^{*} \mathbb{R}^{n}\right)$. The subalgebra $\mathscr{S}\left(T^{*} \mathbb{R}^{n}\right)$ is also stable under the holomorphic functional calculus.

It is well known that $\mathscr{K}^{\infty} \subseteq \mathscr{L}_{1}\left(L^{2}\left(\mathbb{R}^{n}\right)\right)$. Denote by $\operatorname{Tr}$ the restriction of the canonical trace of $\mathscr{K}\left(L^{2}\left(\mathbb{R}^{n}\right)\right)$ to $\mathscr{K}^{\infty}$. For $j=1, \ldots, n$, set $D_{j}=\frac{\partial}{\partial x}$, and let $M_{j}$ denote the pointwise multiplication operator on $\left(L^{2}\left(\mathbb{R}^{n}\right)\right)$ by the coordinate function $x_{j}$. For any $T \in \mathscr{K}^{\infty}$, the commutator $\left[D_{j}, T\right]$ extends to a compact operator, and $\delta_{2 j-1}(T)=\left[D_{j}, T\right]$ defines a derivation $\delta_{2 j-1}: \mathscr{K}^{\infty} \rightarrow \mathscr{K}^{\infty}$. Similarly, $\delta_{2 j}(T)=\left[M_{j}, T\right]$ also defines a derivation of $\mathscr{K}^{\infty}, j=1, \ldots, n$. It is straightforward to check that $\delta_{j} \delta_{k}=\delta_{k} \delta_{j}, j, k=1, \ldots, n$, and that $\operatorname{Tr}\left(\delta_{j}(T)\right)=0$ for any $T \in \mathscr{K}^{\infty}$, $j=1, \ldots, n$. From those observations, it follows that the $(2 n+1)$-linear functional $\omega$ on $\mathscr{K}^{\infty}$ defined by

$$
\omega\left(T_{0}, \ldots, T_{2 n}\right)=\frac{(-1)^{n}}{n !} \sum_{\sigma \in S_{2 n}} \operatorname{sgn}(\sigma) \operatorname{Tr}\left(T_{0} \delta_{\sigma(1)}\left(T_{1}\right) \cdots \delta_{\sigma(2 n)}\left(T_{2 n}\right)\right)
$$

is a cyclic $2 n$-cocycle.

Let $q \in \mathscr{K}^{\infty}$ be a rank one projection. Then

$$
\omega(q, \ldots, q)=1 \text {. }
$$

Since $\mathscr{K}^{\infty}$ is stable under the holomorphic functional calculus, the pairing between the class of $\omega$ and $K_{0}\left(\mathscr{K}\left(L^{2}\left(\mathbb{R}^{n}\right)\right)\right)$ is well defined. By definition ([Co, Proposition 14]),

$$
\left\langle[q],\left[(2 \pi i)^{n} n ! \omega\right]\right\rangle=1 .
$$

Recall the continuous field of $C^{*}$-algebras $\mathscr{A}^{\prime}=\left(A^{\prime}(\hbar)\right)$ introduced in the beginning of this section. For each $\hbar>0, \omega$ defines a densely defined cyclic $2 n$-cocycle 
on $A^{\prime}(\hbar)=\mathscr{K}\left(L^{2}\left(\mathbb{R}^{n}\right)\right)$. We denote by $\varepsilon_{0}$ the cyclic $2 n$-cocycle on $\mathscr{S}\left(T^{*} \mathbb{R}^{n}\right)$ defined by

$$
\varepsilon_{0}\left(f^{0}, \ldots, f^{2 n}\right)=\frac{1}{(2 \pi i)^{n} n !} \int_{T^{*} \mathbb{R}^{n}} f^{0} d f^{1} \wedge \cdots \wedge d f^{n},
$$

where $T^{*} \mathbb{R}^{n}$ is oriented by $d x_{1} \wedge d \xi_{1} \wedge \cdots \wedge d x_{n} \wedge d \xi_{n}>0$.

Let $\hbar \in[0,1]$ be fixed. For $f, g \in \mathscr{S}\left(T^{*} \mathbb{R}^{n} \times \mathbb{R}\right)$, let us define $\hat{f} *_{\hbar} \hat{g}$ by

$$
\left(\hat{f} *_{\hbar} \hat{g}\right)(x, y, \hbar)=(2 \pi)^{-n} \int \hat{f}(x, z, \hbar) \hat{g}(x+\hbar z, y-z, \hbar) d z .
$$

In particular, if $\hbar=0$, then

$$
\left(\hat{f} *_{0} \hat{g}\right)(x, y, 0)=(\widehat{f g})(x, y, 0),
$$

where $f g$ is the product in $C_{0}\left(T^{*} \mathbb{R}^{n}\right)$.

For $\hbar>0$, we have

$$
\rho_{\hbar}(\hat{f}) \rho_{\hbar}(\hat{g})=\rho_{\hbar}\left(\hat{f} *_{\hbar} \hat{g}\right) \quad \text { in } B\left(L^{2}\left(\mathbb{R}^{n}\right)\right) .
$$

By a straightforward computation,

$$
\operatorname{Tr}\left(\rho_{\hbar}(\hat{f})\right)=\frac{1}{\hbar^{n}(2 \pi)^{n}} \int \hat{f}(x, 0, \hbar) d x=\frac{1}{\hbar^{n}(2 \pi)^{n}} \int_{T^{*} \mathbb{R}^{n}} f(x, \xi, \hbar) d x d \xi .
$$

Furthermore,

$$
\begin{gathered}
\delta_{2 j-1}\left(\rho_{\hbar}(\hat{f})\right)=\left[D_{j}, \rho_{\hbar}(\hat{f})\right]=\rho_{\hbar}\left(\left(\frac{\widehat{\partial f}}{\partial x_{j}}\right)\right), \quad j=1, \ldots, n, \\
\delta_{2 j}\left(\rho_{\hbar}(\hat{f})\right)=\left[M_{j}, \rho_{\hbar}(\hat{f})\right]=\rho_{\hbar}\left(-\frac{\hbar}{i}\left(\frac{\widehat{\partial f}}{\partial \xi_{j}}\right)\right), \quad j=1, \ldots, n .
\end{gathered}
$$

It is easy to see that, for given $f_{0}, \ldots, f_{2 n} \in \mathscr{S}\left(T^{*} \mathbb{R}^{n} \times \mathbb{R}\right)$, the function

$$
\hbar \mapsto \omega\left(\rho_{\hbar}\left(\hat{f}_{0}\right), \ldots, \rho_{\hbar}\left(\hat{f}_{2 n}\right)\right.
$$

is continuous on $(0,1]$.

Lemma 5.14. In the limit as $\hbar \rightarrow 0$,

$$
\omega\left(\rho_{\hbar}\left(\hat{f}_{0}\right), \ldots, \rho_{\hbar}\left(\hat{f}_{2 n}\right)\right) \rightarrow \varepsilon_{0}\left(\rho_{0}\left(\hat{f}_{0}\right), \ldots, \rho_{0}\left(\hat{f}_{2 n}\right)\right) .
$$

Proof (see $[\mathrm{N}-\mathrm{N}]$ also). By the definition of $\omega$, we have

$$
\begin{aligned}
& \omega\left(\rho_{\hbar}\left(\hat{f}_{0}\right), \ldots, \rho_{\hbar}\left(\hat{f}_{2 n}\right)\right) \\
& \quad=\frac{(-1)^{n}}{n !} \sum_{\sigma \in S_{2 n}} \operatorname{sgn}(\sigma) \operatorname{Tr}\left(\rho_{\hbar}\left(\hat{f}^{0}\right) \delta_{\sigma(1)}\left(\rho_{\hbar}\left(\hat{f}_{1}\right)\right) \cdots \delta_{\sigma(2 n)}\left(\rho_{\hbar}\left(\hat{f}_{2 n}\right)\right)\right) .
\end{aligned}
$$


Let us analyze the term $\operatorname{Tr}\left(\rho_{\hbar}\left(\hat{f}_{0}\right) \delta_{1}\left(\rho_{\hbar}\left(\hat{f}_{1}\right)\right) \cdots \delta_{2 n}\left(\rho_{\hbar}\left(\hat{f}_{2 n}\right)\right)\right)$. By the observations (5.12) and (5.13),

$$
\begin{aligned}
& \operatorname{Tr}\left(\rho_{\hbar}\left(\hat{f}_{0}\right) \delta_{1}\left(\rho_{\hbar}\left(\hat{f}_{1}\right) \cdots \delta_{2 n}\left(\rho_{\hbar}\left(\hat{f}_{2 n}\right)\right)\right)\right. \\
& =\frac{(-\hbar)^{n}}{i^{n}} \operatorname{Tr}\left(\rho _ { \hbar } \left(\hat{f}_{0} *_{\hbar}\left(\frac{\widehat{\partial f_{1}}}{\partial x_{1}}\right) *_{\hbar}\left(\frac{\widehat{\partial f_{2}}}{\partial \xi_{1}}\right)\right.\right. \\
& \left.\left.*_{\hbar} \cdots *_{\hbar}\left(\frac{\partial \widehat{f_{2 n}-1}}{\partial x_{n}}\right) *_{\hbar}\left(\frac{\widehat{\partial f_{2 n}}}{\partial \xi_{n}}\right)\right)\right) \\
& =\frac{(-1)^{n}}{(2 \pi i)^{n}} \int_{\mathbb{R}^{n}}\left(\hat{f}_{0} *_{\hbar}\left(\frac{\widehat{\partial f_{1}}}{\partial x_{1}}\right) *_{\hbar} \cdots *_{\hbar}\left(\frac{\widehat{\partial f_{2 y}}}{\partial \xi_{n}}\right)\right)(x, 0, \hbar) d x \\
& \rightarrow \frac{(-1)^{n}}{(2 \pi i)^{n}} \int_{T^{*} \mathbb{R}^{n} \times\{0\}} f_{0} \frac{\partial f_{1}}{\partial x_{1}} \frac{\partial f_{2}}{\partial \xi_{1}} \cdots \frac{\partial f_{2 n-1}}{\partial x_{n}} \frac{\partial f_{2 n}}{\partial \xi_{n}} d x d \xi \quad \text { as } \hbar \rightarrow 0 .
\end{aligned}
$$

Similar computations apply to the other terms, with the result that

$$
\begin{aligned}
\lim _{\hbar \rightarrow 0} \omega\left(\rho_{\hbar}\left(\hat{f}_{0}\right), \ldots, \rho_{\hbar}\left(\hat{f}_{2 n}\right)\right) & =\frac{1}{(2 \pi i)^{n} n !} \int_{T^{*} \mathbb{R}^{n} \times\{0\}} f_{0} d f_{1} \wedge \cdots \wedge d f_{2 n} \\
& =\varepsilon\left(\rho_{0}\left(\hat{f_{0}}\right), \ldots, \rho_{0}\left(\hat{f}_{2 n}\right)\right) .
\end{aligned}
$$

Everything we have shown above can be generalized to matrices over $\mathscr{S}\left(T^{*} \mathbb{R}^{n} \times\right.$ $\mathbb{R}$ ). As for cyclic cocycles, it is enough to consider the cup products $\omega \# \operatorname{tr}, \varepsilon_{0} \# \operatorname{tr}$ of $\omega, \varepsilon_{0}$ and the matricial trace tr on matrices.

Lemma 5.15. For $f_{0}, \ldots, f_{2 n} \in M_{d}\left(\mathscr{S}\left(T^{*} \mathbb{R}^{n} \times \mathbb{R}\right)\right)$, we have

$$
\lim _{\hbar \rightarrow 0}(\omega \# \operatorname{tr})\left(\rho_{\hbar}\left(\hat{f}_{0}\right), \ldots, \rho_{\hbar}\left(\hat{f}_{2 n}\right)\right)=\frac{1}{(2 \pi i)^{n} n !} \int_{T^{*} \mathbb{R}^{n} \times\{0\}} \operatorname{tr}\left(f_{0} d f_{1} \wedge \cdots \wedge d f_{2 n}\right) .
$$

Now we are in a position to complete the proof of Theorem 3.1.

Given an elliptic pseudodifferential operator $P$ of order $m>0$, with symbol $a$, denote by $e=\left(e_{\hbar}\right)$ the field of graph projections. Since $\mathscr{S}\left(H_{2 n+1}\right)$ is stable under the holomorphic functional calculus in $C^{*}\left(H_{2 n+1}\right)$, there exists a continuous field $e^{\infty}=$ $\left(e_{\hbar}^{\infty}\right) \in M_{2}\left(M_{d}(\Gamma)^{\sim}\right)$ of projections such that $\left\|e-e^{\infty}\right\|<\varepsilon<1$. So, in particular, $e_{\hbar} \infty \sim e_{\hbar}$ (Murray-von Neumann equivalence).

We have

$$
\begin{aligned}
\operatorname{index} P & =\left\langle\left[e_{1}\right]-\left[\left(\begin{array}{ll}
0 & 0 \\
0 & 1
\end{array}\right)\right],\left[(2 \pi i)^{n} n ! \omega\right]\right\rangle \\
& =\left\langle\left[e_{1}^{\infty}\right]-\left[\left(\begin{array}{ll}
0 & 0 \\
0 & 1
\end{array}\right)\right],\left[(2 \pi i)^{n} n ! \omega\right]\right\rangle
\end{aligned}
$$


and, as $\hbar \mapsto e_{\hbar}^{\infty}$ is norm continuous for $\hbar>0$, and $\mathscr{K}^{\infty}$ is stable under the holomorphic functional calculus,

$$
=\left\langle\left[e_{\hbar}^{\infty}\right]-\left[\left(\begin{array}{ll}
0 & 0 \\
0 & 1
\end{array}\right)\right],\left[(2 \pi i)^{n} n ! \omega\right]\right\rangle \text { for all } \hbar>0 .
$$

Then, by continuity (Lemma 5.15),

$$
(*)=\left\langle\left[e_{0}^{\infty}\right]-\left[\left(\begin{array}{ll}
0 & 0 \\
0 & 1
\end{array}\right)\right],\left[(2 \pi i)^{n} n ! \varepsilon_{0}\right]\right\rangle=\left\langle\left[e_{a}\right]-\left[\left(\begin{array}{ll}
0 & 0 \\
0 & 1
\end{array}\right)\right],\left[(2 \pi i)^{n} n ! \varepsilon_{0}\right]\right\rangle,
$$

since $\mathscr{S}\left(T^{*} \mathbb{R}^{n}\right)$ is holomorphically closed in $C_{0}\left(T^{*} \mathbb{R}^{n}\right)$.

Although $\hat{e}_{0}=e_{0}-\left(\begin{array}{ll}0 & 0 \\ 0 & 1\end{array}\right)$ does belong to the domain of the extension of $\varepsilon_{0}$, we do not know yet that

$$
\left\langle\left[e_{a}\right]-\left[\left(\begin{array}{ll}
0 & 0 \\
0 & 1
\end{array}\right)\right],\left[(2 \pi i)^{n} n ! \varepsilon_{0}\right]\right\rangle=\frac{1}{(2 \pi i)^{n} n !} \int_{T^{*} \mathbb{R}^{n}} \operatorname{tr}\left(\hat{e}_{a}\left(d \hat{e}_{a}\right)^{2 n}\right) .
$$

A problem arises from the fact that in general in a subalgebra $A$ of $C_{0}\left(T^{*} \mathbb{R}^{n}\right)$ such that $\mathscr{S}\left(T^{*} \mathbb{R}^{n}\right) \subseteq A$, and $\hat{e}_{a} \in A$, the classes $\left[e_{a}\right]-\left[\left(\begin{array}{ll}0 & 0 \\ 0 & 1\end{array}\right)\right]$ and $\left[e_{0}^{\infty}\right]-\left[\left(\begin{array}{ll}0 & 0 \\ 0 & 1\end{array}\right)\right]$ may be different in $K_{0}(A)$. To circumvent this difficulty we can argue as follows.

Let $A$ be the subalgebra of $C_{0}\left(T^{*} \mathbb{R}^{n}\right)$ consisting of symbols of order $\leqq-m$. It is straightforward to show that $A$ is stable under the holomorphic functional calculus in $C_{0}\left(T^{*} \mathbb{R}^{n}\right)$. We have $\hat{e}_{a} \in M_{2 d}(A)$. Now, the equality

$$
\left[e_{a}\right]-\left[\left(\begin{array}{ll}
0 & 0 \\
0 & 1
\end{array}\right)\right]=\left[e_{0}^{\infty}\right]-\left[\left(\begin{array}{ll}
0 & 0 \\
0 & 1
\end{array}\right)\right]
$$

holds in $K_{0}(A)$ and $\varepsilon_{0}$ is defined on $A$. The equality (**) above follows.

This finishes the proof of Theorem 3.1.

\section{The Topological Index}

In this section we shall briefly discuss a variant of the topological index formula.

Suppose that $P_{a}$ is an elliptic pseudodifferential operator of arbitrary order, with symbol $a$, acting on $L^{2}\left(\mathbb{R}^{n}, \mathbb{C}^{v}\right)$. So, $a$ is a $v \times v$ matrix valued symbol. For such an operator, the Index $P_{a}$ is well defined [Sh]. Since $a$ is elliptic, there exists $R>0$ such that $a$ is invertible on $|x|^{2}+|\xi|^{2} \geqq R$. Denote the $(2 n-1)$-dimensional sphere $|x|^{2}+|\xi|^{2}=R$ by $S^{2 n-1}$. The restriction of $a$ to $S^{2 n-1}$ is a $G L(v, \mathbb{C})$-valued $C^{\infty}$ function on $S^{2 n-1}$.

Denote by $X$ the outward unit normal vector field on $S^{2 n-1} \subseteq T^{*} \mathbb{R}^{n}$. Let us choose the orientation of $S^{2 n-1}$ so that the $(2 n-1)$-form $l_{X}\left(d x_{1} \wedge d \xi_{1} \wedge \cdots \wedge d x_{n} \wedge\right.$ $\left.d \xi_{n}\right)$ on $S^{2 n-1}$ is positive.

Theorem 6.1 (cf. [H, Thm. 19.3.1], [B-S, Thm. A]). Suppose that a is an elliptic symbol. The following equality holds.

$$
\text { index } P_{a}=\frac{(n-1) !}{(2 \pi i)^{n}(2 n-1) !} \int_{S^{2 n-1}} \operatorname{tr}\left(\left(a^{-1} d a\right)^{2 n-1}\right) .
$$


Proof.

Part 1 The positive order case. We will show that

$$
\frac{(n-1) !}{(2 \pi i)^{n}(2 n-1) !} \int_{S^{2 n-1}} \operatorname{tr}\left(\left(a^{-1} d a\right)^{2 n-1}\right)=\frac{1}{(2 \pi i)^{n} n !} \int_{T^{*} \mathbb{R}^{n}} \operatorname{tr}\left(e_{a}\left(d e_{a}\right)^{2 n}\right) .
$$

In order to do so, we need to recall the description of $K^{0}\left(T^{*} \mathbb{R}^{n}\right)$ in terms of complexes of vector bundles with compact support ([A-S $]$ ).

For any locally compact space $Y$, denote by $\mathbb{C}_{Y}^{v}$ the product bundle $Y \times \mathbb{C}^{v}$ on $Y$. Since $a$ is an isomorphism on $|x|^{2}+|\xi|^{2} \geqq 0$, the triple $\left(\mathbb{C}_{T^{*}}^{v} \mathbb{R}^{n}, \mathbb{C}_{T^{*}}^{v} \mathbb{R}^{n}, a\right)$ is a complex of vector bundles with compact support. Hence we get the class $\left[\mathbb{C}_{T^{*}}^{v} \mathbb{R}^{n}, \mathbb{C}_{T^{*} \mathbb{R}^{n}}^{v}, a\right] \in K^{0}\left(T^{*} \mathbb{R}^{n}\right)$. Let $B_{0}$ and $B_{\infty}$ be closed subsets of $\left(T^{*} \mathbb{R}^{n}\right)^{+}=S^{2 n}$ such that $S^{2 n}=B_{0} \cup B_{\infty}, B_{0} \cap B_{\infty}=S^{2 n-1}$, and $0 \in B_{0}$. By the clutching construction, we obtain a vector bundle $E=\mathbb{C}_{B_{0}}^{v} \cup_{a} \mathbb{C}_{B_{\infty}}^{v}$. When we describe $K_{0}\left(T^{*} \mathbb{R}^{n}\right)$ as difference bundles, the class $[E]-\left[\mathbb{C}_{S^{2 n}}^{v}\right]$ is precisely the class $\left[\mathbb{C}_{T^{*} \mathbb{R}^{n}}^{v}, \mathbb{C}_{T^{*} \mathbb{R}^{n}}^{v}, a\right]$.

Suppose now that $\sigma$ is a $G L(N, \mathbb{C})$-valued $C^{\infty}$-function on $S^{2 n-1}$. Again by the clutching construction we get a vector bundle $\mathbb{C}_{B_{0}}^{N} \cup \mathbb{C}_{B_{\infty}}^{N}$. Then the map $\sigma \mapsto\left[\mathbb{C}_{B_{0}}^{N} \cup\right.$ $\left.\mathbb{C}_{B_{\infty}}^{N}\right]-\left[\mathbb{C}_{S^{2 n}}^{N}\right] \in K^{0}\left(S^{2 n}\right)$ determines a (well defined) map $\delta: K^{1}\left(S^{2 n-1}\right) \rightarrow K^{0}\left(S^{2 n}\right)$, and induces an isomorphism onto $K^{0}\left(T^{*} \mathbb{R}^{n}\right) \subseteq K^{0}\left(S^{2 n}\right)$.

It is not difficult to see that, by the canonical isomorphism

$$
K_{0}\left(C_{0}\left(T^{*} \mathbb{R}^{n}\right)\right) \cong K^{0}\left(T^{*} \mathbb{R}^{n}\right),
$$

the classes $\left[e_{a}\right]-\left[\left(\begin{array}{ll}0 & 0 \\ 0 & 1\end{array}\right)\right]$ and $\left[\mathbb{C}_{B_{0}}^{v} \cup_{a} \mathbb{C}_{B_{\infty}}^{v}\right]-\left[\mathbb{C}_{S^{2 n}}^{v}\right]$ coincide. Thus,

$$
\delta([a])=\left[e_{a}\right]-\left[\left(\begin{array}{ll}
0 & 0 \\
0 & 1
\end{array}\right)\right]
$$

in $K_{0}\left(C_{0}\left(T^{*} \mathbb{R}^{n}\right)\right) \cong K^{0}\left(T^{*} \mathbb{R}^{n}\right)$, where $[a]$ is the class of $a$ in $K^{1}\left(S^{2 n-1}\right) \cong$ $K_{1}\left(C\left(S^{2 n-1}\right)\right)$.

The left-hand side of $(*)$ is the pairing between a $K_{1}$-class $[a]$ and a $(2 n-$ 1 )-trace on $C\left(S^{2 n-1}\right)$, while the right-hand side is the pairing between a $K_{0}$-class $\left[e_{a}\right]-\left[\left(\begin{array}{ll}0 & 0 \\ 0 & 1\end{array}\right)\right]$ and a $2 n$-trace on $C_{0}\left(T^{*} \mathbb{R}^{n}\right)$. Since $K_{1}\left(C\left(S^{2 n-1}\right)\right)$ is singly generated, in order to complete the proof of Theorem 6.1, we have only to exhibit an elliptic operator $P$ of positive order with index $P=1$ and to show that the left-hand side of $(*)$ takes value 1 for such a $P$.

We construct an $M_{2 j-1}(\mathbb{C})$-valued symbol $a_{j}$ on $\mathbb{R}^{j}$ by induction. Set $a_{1}\left(x_{1}, \xi_{1}\right)=$ $x_{1}+i \xi_{1}$. Suppose that $a_{j-1}\left(x_{1}, \ldots, x_{j-1}, \xi_{1}, \ldots, \xi_{j-1}\right)$ is defined. Define $a_{j}$ by

$$
\begin{aligned}
& a_{j}\left(x_{1}, \ldots, x_{j}, \xi_{1}, \ldots, \xi_{j}\right) \\
& \quad=\left(\begin{array}{cc}
a_{j-1}\left(x_{1}, \ldots, x_{j-1}, \xi_{1}, \ldots, \xi_{j-1}\right) & -\overline{\left(x_{i}+i \xi_{j}\right)} I \\
\left(x_{j}+i \xi_{j}\right) I & a_{j-1}\left(x_{1}, \ldots, x_{j-1}, \xi_{1}, \ldots, \xi_{j-1}\right)
\end{array}\right),
\end{aligned}
$$

where $I$ is the $2^{j-2} \times 2^{j-2}$ identity matrix (cf. [V]).

It is clear that $a_{j}$ is elliptic, of order 1 . Notice that $P_{a_{1}}=x+\frac{d}{d x}$. This is a standard construction of elliptic operators on higher-dimensional spaces. More precisely, $P_{a_{n}}$ is the tensor product $P_{a_{n}}=P_{a_{1}} \# \ldots \# P_{a_{1}}$ of $n$ copies of $P_{a_{1}}$ (see, e.g., [B-B]). 
Hence, index $P_{a_{n}}=1$. Therefore, the right-hand side of $(*)$ is equal to 1 by Theorem 3.1. A straightforward computation shows that the left-hand side of $(*)$ is also equal to 1 . Thus, the equality $(*)$ holds for arbitrary elliptic symbols of positive order, and Theorem 6.1 is proved in this case.

Part 2. The nonpositive order case. Suppose that $P$ is elliptic of order $m \leqq 0$. Let $l$ be a positive integer such that $2 l+m>0$. Choose an elliptic operator $\wedge_{0}$ of order $l$, and consider $\wedge=\wedge_{0}^{*} \wedge_{0}$. Choose $R>0$ sufficiently large that the (left) symbols $\sigma(\wedge), \sigma(\wedge P), \sigma(P)$ are invertible on $|x|^{2}+|\xi|^{2} \geqq R^{2}$. Since $\wedge$ is formally self-adjoint, index $(\wedge)=0$. Hence, index $(P)=\operatorname{index}(\wedge P)$.

It is easy to see that, by asymptotic expansion,

$$
\sigma(\wedge P)-\sigma(\wedge) \sigma(P) \in \Gamma^{2 l+m-2}
$$

From this it follows that $\sigma(\wedge P), \sigma(\wedge) \sigma(P)$ are homotopic on the sphere $S^{2 n-1}=$ $\left\{(x, \xi):|x|^{2}+|\xi|^{2}=R^{2}\right\}$. Therefore, in $K^{1}\left(S^{2 n-1}\right)$, we have the equality

$$
[\sigma(\wedge P)]=[\sigma(\wedge)]+[\sigma(P)]
$$

By the choice of $\wedge$, we obtain an asymptotic expansion of $\sigma(\wedge)$ such that the leading term $\sigma_{0}(\wedge)$ is self-adjoint, and $\sigma(\wedge)-\sigma_{0}(\wedge) \in \Gamma^{2 l-2}$. Hence, $\sigma(\wedge)$ and $\sigma_{0}(\wedge)$ are homotopic, and

$$
[\sigma(\wedge)]=\left[\sigma_{0}(\wedge)\right]=0
$$

in $K^{1}\left(S^{2 n-1}\right)$. Therefore,

$$
[\sigma(P)]=[\sigma(\wedge P)] \in K^{1}\left(S^{2 n-1}\right)
$$

Hence, by Part 1 of the proof,

$$
\begin{aligned}
\operatorname{index}(P) & =\operatorname{index}(\wedge P)=\frac{(n-1) !}{(2 \pi i)^{n}(2 n-1) !} \int_{S^{2 n-1}} \operatorname{tr}\left(\left(\sigma(\wedge P)^{-1} d \sigma(\wedge P)\right)^{2 n-1}\right) \\
& =\frac{(n-1) !}{(2 \pi i)^{n}(2 n-1) !} \int_{S^{2 n-1}} \operatorname{tr}\left(\left(\sigma(P)^{-1} d \sigma(P)\right)^{2 n-1}\right) .
\end{aligned}
$$

Thus, the formula given in Theorem 6.1 is valid also for elliptic operators of non-positive order.

The topological index formula in Theorem 6.1 is identical to the formulae in $[\mathrm{B}-\mathrm{S}]$ and $[\mathrm{H}]$ except for sign. This difference comes from the different choices of the orientation on $S^{2 n-1}$.

Acknowledgements This work was supported by research grants from the Natural Sciences and Engineering Research Council of Canada, the Danish Natural Sciences Research Council, and the United States National Science Foundation. 


\section{References}

[A-S] Atiyah, M F, Singer, I M : The index of elliptic operators I Ann of Math 87, 484-530 (1968)

[B-B] Booss, B , Bleecker, D D : Topology and Analysis: The Atiyah-Singer Index Formula and Gauge-Theoretic Physics Universitext, Berlin-Heidelberg-New York: Springer-Verlag, 1985

[B-S] Bott, R, Seeley, R : Some remarks on the paper of Callias. Commun Math. Phys 62, 235-245 (1978)

[Ca] Callias, C: Aximal anomalies and index theorem on open spaces Commun Math Phys 62, 213-234 (1978)

[Co] Connes, A : Non-commutative differential geometry, Parts I and II Inst Hautes Études Sci Publ Math 62, 257--360 (1985)

[D] Dixmier, J : $C^{*}$-algebras and their representations Paris: Gauthier-Villars, 1964

[E-N-N] Elliott, G A, Natsume, T, Nest, R.: The Heisenberg group and $K$-theory $K$-Theory 7 , 409-428 (1993)

[H] Hörmander, L: The Analysis of Linear Partial Differential Operators III Grundlehren der mathematischen Wissenschaften 274, Berlin-Heidelberg-New York: Springer-Verlag, 1985

[K] Kumano-go, H: Pseudo-differential Operators. Cambridge, Massachusetts: MIT Press, 1981

[N-N] Natsume, T, Nest, R: The local structure of the cyclic cohomology of Heisenberg Lie groups. J Funct Anal 119, 481-498 (1994)

[R] Rieffel, M A : Continuous fields of $C^{*}$-algebras coming from group cocycles and actions Math Ann 283, 631-643 (1989)

[S] Seeley, R: Complex powers of an elliptic operator Proc Symp Pure Math 10, 288-307 (1967)

[Sh] Shubin, M A : Pseudodifferential Operators and Spectral Theory Tokyo: Springer-Verlag, 1987

[T] Taylor, M: Pseudodifferential Operators Princeton, NJ: Princeton University Press, 1981

[V] Venugopalkrishna, U: Fredholm operators associated with strongly pseudoconvex domains in $\mathbb{C}^{n}$ J Funct Anal 9, 349-373 (1972)

Communicated by $\mathrm{H}$ Araki 
